\title{
Hierarchical Control of Thermostatically Controller Loads for Primary Frequency
} Control

Zhao, Haoran; Wu, Qiuwei; Huang, Shaojun; Zhang, Hengxu; Xue, Yusheng

Published in:

IEEE Transactions on Smart Grid

Link to article, DOI:

10.1109/TSG.2016.2624509

Publication date:

2018

Document Version

Peer reviewed version

Link back to DTU Orbit

Citation (APA):

Zhao, H., Wu, Q., Huang, S., Zhang, H., \& Xue, Y. (2018). Hierarchical Control of Thermostatically Controller Loads for Primary Frequency Control. IEEE Transactions on Smart Grid, 9(4), 2986-2998.

https://doi.org/10.1109/TSG.2016.2624509

\section{General rights}

Copyright and moral rights for the publications made accessible in the public portal are retained by the authors and/or other copyright owners and it is a condition of accessing publications that users recognise and abide by the legal requirements associated with these rights.

- Users may download and print one copy of any publication from the public portal for the purpose of private study or research.

- You may not further distribute the material or use it for any profit-making activity or commercial gain

- You may freely distribute the URL identifying the publication in the public portal 


\title{
Hierarchical Control of Thermostatically Controlled Loads for Primary Frequency Support
}

\author{
Haoran Zhao, Qiuwei Wu, Shaojun Huang, Hengxu Zhang, Yutian Liu and Yusheng Xue
}

\begin{abstract}
This paper proposes a hierarchical control of Thermostatically Controlled Loads (TCLs) to provide primary frequency control support. The control architecture is comprised of three levels. At the high level, an aggregator coordinates multiple distribution substations and dispatches the primary reserve references. At the middle level, distribution substations estimate the available power of TCLs based on the aggregated bin model, and dispatch control signals to individual TCLs. At the local level, a supplementary frequency control loop is implemented at the local controller, which makes TCLs respond to the frequency event autonomously. Case studies show that the proposed controller can efficiently respond to frequency events and fulfill the requirement specified by the system operator. The users' comforts are not compromised and the short cycling of TCLs is largely reduced. Due to the autonomous control, the communication requirement is minimized.
\end{abstract}

Index Terms-Markov transition matrix, primary frequency support, thermostatically controlled loads.

\section{NOMENCLATURE}

A. Individual TCL Model

$\begin{array}{ll}C & \text { TCL thermal capacitance }\left[\mathrm{kWh} /{ }^{\circ} \mathrm{C}\right] . \\ R & \text { TCL thermal resistance }\left[{ }^{\circ} \mathrm{C} / \mathrm{kW}\right] . \\ P_{\text {rate }} & \text { TCL rated power }[\mathrm{kW}] . \\ \text { COP } & \text { Coefficient of performance }[-] . \\ T & \text { Temperature }\left[{ }^{\circ} \mathrm{C}\right] . \\ T_{\mathrm{a}} & \text { Ambient temperature }\left[{ }^{\circ} \mathrm{C}\right] . \\ T_{\text {set }} & \text { Temperature reference }\left[{ }^{\circ} \mathrm{C}\right] . \\ \delta_{\mathrm{db}} & \text { Temperature dead-band width }\left[{ }^{\circ} \mathrm{C}\right] . \\ m & \text { Switch control signal ON/OFF }[-] . \\ \omega & \text { Process noise of TCL }\left[{ }^{\circ} \mathrm{C}\right] .\end{array}$

\section{B. Aggregated TCL Model}

$\begin{array}{ll}\boldsymbol{A} & \text { State space matrix [-]. } \\ \boldsymbol{B} & \text { State space matrix [-]. } \\ \boldsymbol{C} & \text { State space matrix [-]. } \\ N_{\mathrm{b}} & \text { Number of bins [-]. } \\ T_{\mathrm{n}} & \text { Normalized temperature [-]. } \\ N_{\mathrm{tcl}} & \text { Number of TCLs [-]. }\end{array}$

H. Zhao and S. Huang are with the Center for Electric Power and Energy (CEE), Department of Electrical Engineering, Technical University of Denmark (DTU), Kgs. Lyngby, 2800 Denmark (e-mail: hzhao@elektro.dtu.dk).

Q. Wu is with the Center for Electric and Energy (CEE), Department of Electrical Engineering, Technical University of Denmark (DTU), Kgs. Lyngby, 2800 Denmark and School of Electrical Engineering, Shandong University, China (e-mail: qw@elektro.dtu.dk).

H. Zhang and Y. Liu are with the Key Laboratory of Power System Intelligent Dispatch and Control, of the Ministry of Education (Shandong University), Jinan 250061, China (e-mail: zhanghx@sdu.edu.cn, liuyt@sdu.edu.cn).

Y. Xue is with the State Grid Electric Power Research Institute, Nanjing 210003, China (e-mail: xueyusheng@sgepri.sgcc.com.cn).

$\begin{array}{ll}\bar{P} & \begin{array}{l}\text { Mean power consumption of the TCLs } \\ {[\mathrm{kW}] .}\end{array} \\ P_{\text {total }} & \text { Aggregated power }[\mathrm{kW}] . \\ \boldsymbol{x} & \text { State bin vector }[-] . \\ \boldsymbol{x}_{\text {meas }} & \text { Measured state bin vector }[-] . \\ \boldsymbol{u} & \text { Control input vector }[-] . \\ \boldsymbol{y} & \text { Output vector }[-] .\end{array}$

C. Frequency Droop Control
$f_{\mathrm{m}}$
$f_{\text {ref }}$
$\Delta f$
$\Delta f_{\mathrm{db}}$
$\Delta f_{\max }$
$f_{\max }, f_{\min }$
Frequency measurement $[\mathrm{Hz}]$.
Frequency reference $[\mathrm{Hz}]$.
Frequency deviation to the nominal value $[\mathrm{Hz}]$.
Frequency dead-band width [Hz].
Maximum frequency deviation $[\mathrm{Hz}]$.
Maximum and minimum frequencies $[\mathrm{Hz}]$.

\section{Hierarchical Control}

\begin{tabular}{|c|c|}
\hline$N_{\mathrm{S}}$ & Number of distribution substations [-]. \\
\hline$P_{\text {prim }}^{\mathrm{up}}, P_{\text {prim }}^{\mathrm{dn}}$ & $\begin{array}{l}\text { Primary reserve references for up and } \\
\text { down regulation }[\mathrm{kW}] \text {. }\end{array}$ \\
\hline$P_{\text {avi }}^{\mathrm{up}}, P_{\mathrm{avi}}^{\mathrm{dn}}$ & $\begin{array}{l}\text { Estimated available power reserves for up } \\
\text { and down regulation }[\mathrm{kW}] \text {. }\end{array}$ \\
\hline$N_{\text {sta }}^{\text {on }}, N_{\text {end }}^{\text {on }}$ & $\begin{array}{l}\text { Starting and ending indices of the feasible } \\
\text { ON bins [-]. }\end{array}$ \\
\hline$N_{\text {sta }}^{\text {off }}, N_{\text {end }}^{\text {off }}$ & $\begin{array}{l}\text { Starting and ending indices of the feasible } \\
\text { OFF bins [-]. }\end{array}$ \\
\hline$N_{\mathrm{p}}$ & Prediction number $[-]$. \\
\hline$\alpha$ & Transition rate from $\mathrm{ON}$ to $\mathrm{OFF}[-]$. \\
\hline$\beta$ & Transition rate from OFF to $\mathrm{ON}[-]$. \\
\hline$\alpha_{\text {set }}$ & $\begin{array}{l}\text { Predefined threshold of the transition rate } \\
\text { from ON to OFF [-]. }\end{array}$ \\
\hline$\beta_{\text {set }}$ & $\begin{array}{l}\text { Predefined threshold of the transition rate } \\
\text { from OFF to ON [-] }\end{array}$ \\
\hline$f_{\text {trig }}^{\text {over }}, f_{\text {trig }}^{\text {under }}$ & $\begin{array}{l}\text { Trigger values for over and under frequen- } \\
\text { cies }[\mathrm{Hz}] \text {. }\end{array}$ \\
\hline$N_{\text {c_on }}, N_{\text {c_off }}$ & $\begin{array}{l}\text { Group numbers for } \mathrm{ON} \text { and OFF TCLs } \\
{[-] \text {. }}\end{array}$ \\
\hline$G_{\text {ind }}$ & Group index $[-]$. \\
\hline$K_{\text {ord }}$ & Merit order in the feasible bins [-]. \\
\hline Ctr1, Ctr2, Ctr3 & $\begin{array}{l}\text { Selection criterion for selectors in local } \\
\text { controller [-]. }\end{array}$ \\
\hline$S_{1}, S_{2}$ & Outputs of selectors in local controller [-]. \\
\hline$t_{\mathrm{s}}$ & Control sampling time $[\mathrm{s}]$. \\
\hline$t_{\mathrm{m}}$ & Measurement sampling time $[\mathrm{s}]$. \\
\hline$\Delta t$ & Time delay $[\mathrm{s}]$. \\
\hline
\end{tabular}




\section{E. Kalman filter}

$\begin{array}{ll}\boldsymbol{w}, \boldsymbol{v} & \text { Process and measurement noise [-]. } \\ \boldsymbol{Q}_{\mathbf{k f}} & \text { Covariance matrix of } \boldsymbol{w}[-] . \\ \boldsymbol{R}_{\mathbf{k f}} & \text { Covariance matrix of } \boldsymbol{v}[-] . \\ \boldsymbol{K} & \text { Optimal Kalman gain [-]. } \\ \hat{\boldsymbol{x}}^{-}, \hat{\boldsymbol{x}} & \text { Predicted (priori) and updated (posteriori) } \\ & \text { state estimate [-]. } \\ \boldsymbol{P}^{-}, \boldsymbol{P} & \text { Predicted (priori) and updated (posteriori) } \\ & \text { state estimate covariance [-]. }\end{array}$

\section{F. Simulation}

$\begin{array}{ll}P_{\mathrm{tcl}}^{\mathrm{res}} & \begin{array}{l}\text { Power response of TCLs to the frequency } \\ \text { event [kW]. }\end{array} \\ n_{\mathrm{switch}} & \text { Total switching number [-]. } \\ n_{\mathrm{tcl}} & \text { Number of TCLs, which respond to the } \\ & \text { frequency event [-]. } \\ \eta & \text { Switching times per TCL [-]. }\end{array}$

\section{INTRODUCTION}

$\mathbf{I}$ $\mathrm{N}$ power system operation, maintaining the balance between power generation and consumption is a key issue. As the indicator of the balance, the system frequency needs to be kept within certain limits [1]. To ensure this, the system operator should be capable of providing sufficient frequency control reserve [2]. In the ENTSO-E interconnection, the control reserves are classified into three categories: primary, secondary and tertiary control [3]. Among them, the primary control acts fast and must be activated and respond within a short period (30 seconds). Conventionally, it is realized by speed governor control of synchronous generators.

Nowadays, the integration of large-scale Renewable Energy Sources (RESs) into the power system has considerable impacts on the system operation and introduces technical challenges [4]. Due to the intermittency of RESs, more primary reserves are required. Since the price of the conventional primary reserves is quite high (8,000-22,000 €/MW/year in Denmark), it's not cost-effective to solely rely on the generation side. Alternatively, the demand side can provide reserves to improve system reliability and reduce the cost [5]-[7]. The major obstacles of the demand utilization include the complexity of real-time monitoring for a large number of distributed loads, customer comforts, wear and tear, etc. [8].

Thermostatically Controlled Loads (TCLs), such as space heaters, air conditioners, refrigerators and electric water heaters, account for a large portion of electrical demand [9]. By local hysteresis control, the temperature is kept within a dead-band [10]. The TCLs have inherent operation flexibility. Their power consumption can be switched on and off in time within limits, without compromising the customer comfort [11]. Moreover, the switching action happens almost instantaneously which enables them to respond faster than most conventional generators. These characteristics make TCLs suitable for providing primary frequency reserve. With the expansion of grid cyber-infrastructure, the response of large aggregation of electric loads can be controlled at a level of precision comparable to conventional generators [12].
Several studies have investigated the potential of TCLs to provide frequency reserve. The bin transition modeling technique was proposed to describe the aggregated dynamic behaviour of the TCL population in [6], [12]. The aggregated power is controlled to track the power reference. The central controller directly distributes the ON/OFF commands to TCLs. To realize a fast response to a frequency deviation, near real-time communication infrastructure from an aggregator to TCLs is required. In [8], [13], by installing a frequency sensor and appropriate control intelligence, TCLs can respond autonomously to frequency variation and provide fast reserve to the system. However, the frequency thresholds are fixed. In [14], the frequency thresholds are updated for each control period in order to fulfil the requirement of primary frequency control specified by the system operator. However, for some TCLs, such as air conditioners, the time delay relay are often installed in the compressor to prevent short cycling of the device. The short cycling has not been considered.

This paper aims to manage a portfolio of TCLs to provide primary reserve, which complies with the requirements specified by the system operator. The main contributions are threefold. Firstly, a hierarchical control structure is designed to coordinate the TCLs connected to different substations. Secondly, instead of real-time control, the control cycle is set as a longer period. The transposed Markov Transition Matrix (MTM) is used to estimate the dynamic behaviour during the control interval. Thirdly, a supplemental control loop is proposed on top of the local hysteresis controller. Without compromising the customer comfort, the TCL devices can respond to both over and under frequency events autonomously, with the short cycling largely reduced.

The paper is organized as follows: the modeling of individual and aggregated TCLs is introduced in Section II. Section III describes the requirement of primary frequency control specified by ENTSO-E. The hierarchical control architecture is presented in Section IV. The control implementations at the middle and local levels are described in Section $\mathrm{V}$ and VI, respectively. Simulations are presented and discussed in Section VII, followed by conclusions.

\section{Modeling OF TCLS}

\section{A. Individual TCL Model}

In this study, the individual TCL is modeled by a discrete time difference equation, which is commonly applied to describe the thermodynamic [15],

$$
T(k+1)=a T(k)+(1-a)\left(T_{\mathrm{a}}(k)-m(k) T_{\mathrm{g}}\right)+\omega(k)
$$

with $a \triangleq e^{-\frac{\Delta t}{C R}}$ and

$$
T_{\mathrm{g}} \triangleq \begin{cases}R \cdot \mathrm{COP} \cdot P_{\text {rate }}, & \text { for cooling devices, } \\ -R \cdot \mathrm{COP} \cdot P_{\text {rate }}, & \text { for heating devices, }\end{cases}
$$

where $C$ and $R$ are the thermal capacitance and resistance of TCL, respectively, $\Delta t$ is the time step size, $k$ is the step index, $T$ is the measured temperature, $T_{\mathrm{a}}$ is the ambient temperature, $\omega$ is the noise process, $P_{\text {rate }}$ is the rated power of TCL, $m$ is the switch control variable of TCL, and COP is the coefficient of performance. 
The local hysteresis temperature control with a dead-band is also integrated in the model. The control variable $m$ is determined according to the following rules for heating TCL devices. For cooling devices, the switch status is opposite.

$m(k+1)= \begin{cases}0, & \text { if } T(k+1)>T_{\mathrm{set}}+\frac{\delta_{\mathrm{db}}}{2}: \mathrm{OFF}, \\ 1, & \text { if } T(k+1)<T_{\mathrm{set}}-\frac{\delta_{\mathrm{db}}}{2}: \mathrm{ON}, \\ m(k), & \text { otherwise. }\end{cases}$

where $T_{\text {set }}$ is the temperature reference and $\delta_{\mathrm{db}}$ is the deadband width.

\section{B. Aggregated TCL Model}

The aggregated TCL model proposed in [12] can efficiently describe the dynamics of a heterogeneous TCL population. The state bin transition of a heating TCL is shown in Fig. 1. The temperature dead-band $\delta_{\mathrm{db}}$ is divided into $\frac{N_{\mathrm{b}}}{2}$ intervals. Each interval is further divided into two state bins based on its switch status (ON/OFF). Therefore, a set of $N_{\mathrm{b}}$ state bins can be defined to describe the whole temperature transition of the individual TCL. It is similar for a cooling TCL device.

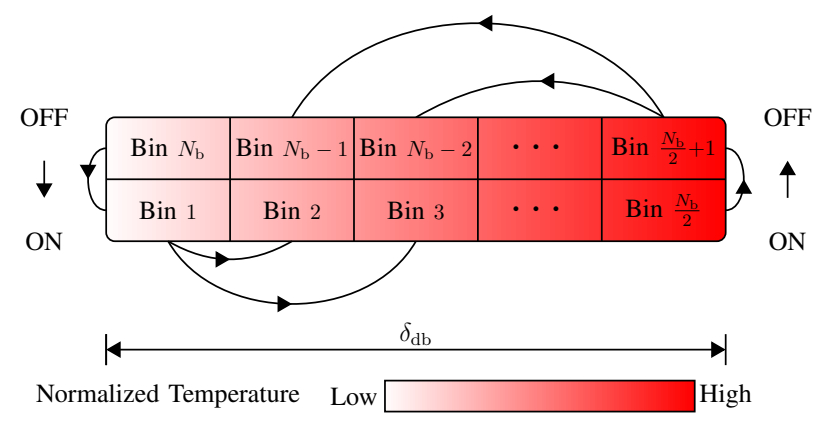

Fig. 1. State bin transition model for a heating TCL

As different devices have different $T_{\mathrm{set}}$ and $\delta_{\mathrm{db}}, \delta_{\mathrm{db}}$ needs to be normalized. The normalized temperature $T_{\mathrm{n}}$ can be derived by,

$$
T_{\mathrm{n}}=\frac{T-\left(T_{\mathrm{set}}-0.5 \delta_{\mathrm{db}}\right)}{\delta_{\mathrm{db}}}, T_{\mathrm{n}} \in[0,1] .
$$

The current bin of a TCL can be decided according to $\left(T_{\mathrm{n}}, m\right)$. A population of TCLs can be aggregated within the bin transition model and expressed in the following state space form,

$$
\begin{aligned}
\boldsymbol{x}(k+1) & =\boldsymbol{A} \boldsymbol{x}(k)+\boldsymbol{B} \boldsymbol{u}(k), \\
\boldsymbol{y}(k) & =\boldsymbol{C} \boldsymbol{x}(k),
\end{aligned}
$$

where $\boldsymbol{x} \in \mathbb{R}^{N_{\mathrm{b}} \times 1}$ is a vector of probability mass, equivalent to the fraction of TCLs in each state bin, and $\boldsymbol{u} \in \mathbb{R}^{\frac{N_{\mathrm{b}}}{2} \times 1}$ is the control vector which affects the switch states of TCLs. Positive elements of $\boldsymbol{u}$ switch TCLs ON, while negative elements of $\boldsymbol{u}$ switch TCLs OFF.

$\boldsymbol{A} \in \mathbb{R}^{N_{\mathrm{b}} \times N_{\mathrm{b}}}$ can be considered as a transposed Markov Transition Matrix (MTM) which describes the transition probability of TCLs from one bin to the next. As the controlled TCLs switch from ON bins to the corresponding OFF bin (vice versa), $\boldsymbol{B} \in \mathbb{R}^{N_{\mathrm{b}} \times \frac{N_{\mathrm{b}}}{2}}$ is structured as follows,

$$
\boldsymbol{B}=\left[\begin{array}{ccc}
1 & & 0 \\
& \ddots & \\
0 & & 1 \\
\hline 0 & & -1 \\
& . & \\
-1 & & 0
\end{array}\right] .
$$

The definition of $C$ is dependent on whether the state information is available in real time. If only the real-time measurement of the aggregated power of TCLs, $P_{\text {total }}$, is available, $\boldsymbol{y}$ is a scalar and $\boldsymbol{C} \in \mathbb{R}^{1 \times N_{\mathrm{b}}}$ is structured as follows,

$$
\boldsymbol{C}=\bar{P} N_{\mathrm{tcl}}[\underbrace{1, \cdots, 1}_{\frac{N_{\mathrm{b}}}{2}}, \underbrace{0, \cdots, 0}_{\frac{N_{\mathrm{b}}}{2}}],
$$

where $\bar{P}$ indicates the mean power consumption of the TCLs and $N_{\text {tcl }}$ is the number of the TCLs. If all state information of all TCLs is available, $\boldsymbol{y} \in \mathbb{R}^{\left(N_{\mathrm{b}}+1\right) \times 1}$ and $\boldsymbol{C} \in \mathbb{R}^{\left(N_{\mathrm{b}}+1\right) \times N_{\mathrm{b}}}$ is structured as follows,

$$
\boldsymbol{C}=\left[\begin{array}{cc}
\frac{\boldsymbol{I}}{\bar{P} N_{\mathrm{tcl}}, \cdots, \bar{P} N_{\mathrm{tcl}}}, & \underbrace{0, \cdots, 0}_{\frac{N_{\mathrm{b}}}{2}}
\end{array},\right.
$$

where $\boldsymbol{I} \in \mathbb{R}^{N_{\mathrm{b}} \times N_{\mathrm{b}}}$ is the identity matrix.

\section{Primary Frequency Control Requirement}

The primary frequency control droop is illustrated in Fig. 2. The up or down regulation will be activated if the measured frequency deviation $\Delta f$ is beyond the dead-band $\Delta f_{\mathrm{db}}$ and reach to its full value at $\Delta f_{\max }$. Within the ranges $\left[\Delta f_{\mathrm{db}}, \Delta f_{\max }\right]$ and $\left[-\Delta f_{\max },-\Delta f_{\mathrm{db}}\right]$, the delivered power is proportional to $\Delta f$. In the ENTSO-E, the parameters of this droop curve are $\Delta f_{\mathrm{db}}=0.02 \mathrm{~Hz}$ and $\Delta f_{\max }=0.2 \mathrm{~Hz}$.

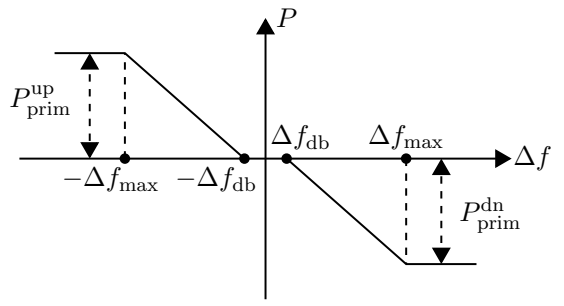

Fig. 2. Primary frequency control droop

\section{Hierarchical Control Architecture}

The proposed hierarchical control architecture is shown in Fig. 3. It consists of three levels. The assumptions of each control level are summarized in Table I.

At the high level, the aggregator is regarded as an entity to participate in the primary reserve market. Ideally, the aggregator would have direct access to each TCL. Due to the large number of TCLs and their wide distribution in areas, it is more efficient to have a middle level, the distribution substation, 


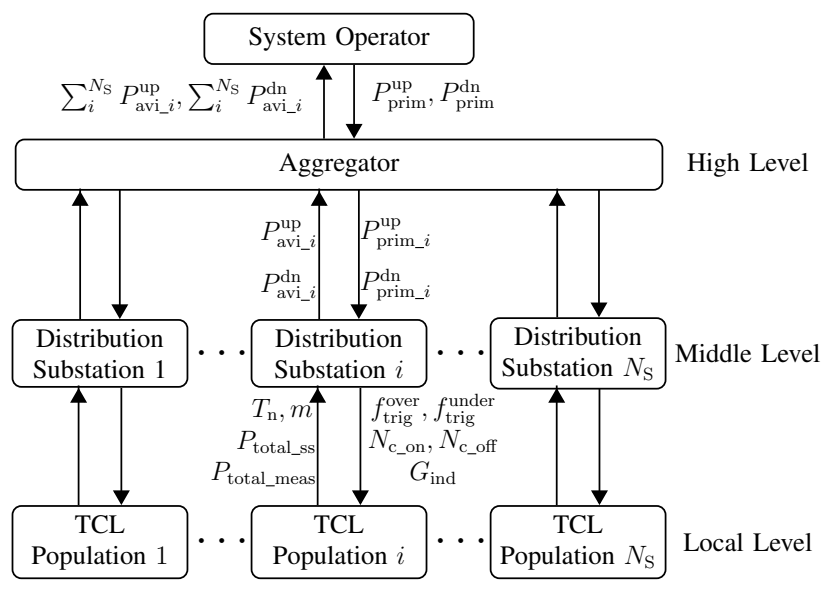

Fig. 3. Proposed hierarchical control structure

TABLE I

ASSUMPTIONS OF EACH CONTROLLER LEVEL

\begin{tabular}{c|l}
\hline \hline Control level & Assumptions \\
\hline \hline Aggregator & $P_{\text {prim }}^{\text {up }} \leq \sum_{i}^{N_{\mathrm{S}}} P_{\text {avi_i } i}^{\text {up }}, P_{\text {prim }}^{\text {dn }} \leq \sum_{i}^{N_{\mathrm{S}}} P_{\text {avi_i } i}^{\text {dn }}$ \\
\hline Distribution & Bidirectional communication with \\
Substation & individual TCLs \\
\hline \multirow{3}{*}{ TCL } & Temperature sensor \\
& ON/OFF state measurement \\
& Frequency measurement \\
\hline
\end{tabular}

to collect the information of individual TCLs within its area through the temperature and power sensors.

By collecting the estimated available reserves $\left(P_{\mathrm{avi} i}^{\mathrm{up}}\right.$ and $\left.P_{\mathrm{avi} i}^{\mathrm{dn}} i\right)$ from the multiple $\left(N_{\mathrm{S}}\right)$ distribution substations, the aggregator delivers their sums $\left(\sum_{i}^{N_{\mathrm{S}}} P_{\mathrm{avi} i}^{\mathrm{up}}\right.$ and $\left.\sum_{i}^{N_{\mathrm{S}}} P_{\mathrm{avi} i}^{\mathrm{dn}}\right)$ to the system operator and compete with the conventional generators. After bidding and approval by the system operator, the aggregator receives the primary reserves cleared for both up and down regulations $\left(P_{\mathrm{prim}}^{\mathrm{up}}\right.$ and $\left.P_{\mathrm{prim}}^{\mathrm{dn}}\right)$. Normally, in order to guarantee that the aggregator has sufficient capacity, $P_{\text {prim }}^{\mathrm{up}} \leq \sum_{i}^{N_{\mathrm{S}}} P_{\mathrm{avi} i}^{\mathrm{up}}$, and $P_{\mathrm{prim}}^{\mathrm{dn}} \leq \sum_{i}^{N_{\mathrm{S}}} P_{\mathrm{avi} i}^{\mathrm{dn}}$. Similar to the droop settings of conventional generators, the primary reserve references $\left(P_{\mathrm{prim}_{-} i}^{\mathrm{up}}\right.$ and $\left.P_{\mathrm{prrim}_{i} i}^{\mathrm{dn}}\right)$ are dispatched proportionally according to $P_{\text {avi } \_i}^{\mathrm{up} i}$ and $P_{\text {avi } \_i}^{\mathrm{dn}}$,

$$
\begin{aligned}
P_{\text {prim } \_i}^{\mathrm{up}} & =\frac{P_{\mathrm{avi} i}^{\mathrm{up}}}{\sum_{j=1}^{N_{\mathrm{s}}} P_{\mathrm{avi} \_}^{\mathrm{up}}} P_{\mathrm{prim}}^{\mathrm{up}}, \\
P_{\mathrm{prim} \_i}^{\mathrm{dn}}= & \frac{P_{\mathrm{avi} i}^{\mathrm{dn}} i}{\sum_{j=1}^{N_{\mathrm{s}}} P_{\mathrm{avi} \_}^{\mathrm{dn}}} P_{\mathrm{prim}}^{\mathrm{dn}} .
\end{aligned}
$$

At the middle level, the state measurments of TCLs including temperature $T_{\mathrm{n}}$ and switch status $m$ are collected and delivered to the distribution substation. Due to the data transmission constraints, higher frequency communication implies more significant investments in grid cyber-infrastructure [11], [16]. In this study, a long control sampling time $t_{\mathrm{s}}$ is adopted (2 minutes or longer), which can significantly reduce these costs. During the control interval, the states of the corresponding TCL population are estimated based on the aggregated model described in Section II-B.
The tasks of the distribution substation are twofold. Firstly, $P_{\mathrm{avi} \_i}^{\mathrm{dn}}$ and $P_{\mathrm{avi} i}^{\mathrm{up}}$ are estimated. $P_{\mathrm{avi} i}^{\mathrm{dn}}$ and $P_{\mathrm{avi} i}^{\mathrm{up}}$ represents the accumulated capacity of OFF and ON devices, respectively, which can be switched during the control interval if required. The estimation algorithm is described in Section V. Secondly, based on the estimated capacities, the over and under frequency references $\left(f_{\text {trig }}^{\text {over }}, f_{\text {trig }}^{\text {under }}\right)$ are calculated and delivered to the local TCL controller. The references are used as the thresholds of the supplemental loop of the local TCL controller. Besides, the ON and OFF devices are grouped. The total group numbers $\left(N_{\mathrm{c}_{-} \text {on }}\right.$ and $\left.N_{\mathrm{c} \_ \text {off }}\right)$ and the group index $\left(G_{\text {ind }}\right)$ are calculated and delivered as well, which are used to manage the switching in a sequential way to avoid the short cycling of TCLs. The implementation is explained in Section VI.

At the local level, the devices are assumed to be able to measure the system frequency. Based on the proposed frequency control loop with derived trigger frequencies $\left(f_{\text {trig }}^{\text {over }}, f_{\text {trig }}^{\text {under }}\right)$, group numbers $\left(N_{\text {c_on }_{\text {on }}}\right.$ and $\left.N_{\text {c_off }}\right)$ and index $\left(G_{\text {ind }}\right)$, the TCLs can respond to the fast frequency events autonomously without communication. Moreover, the customers' comfort is not compromised and the short cycling is largely reduced. More details are described in Section VI.

This study is the follow-up of the research project-Demand as Frequency-controlled Reserve [17]. In that project, a demand response device, referred to as the "SmartBox", has been developed, which has the ability to regulate the connected appliance (see Fig. 4). The relevant data, including temperature and power, can be measured and the data can be communicated with a central server by the mobile network (GSM-based). The SmartBox has been applied in the demonstration in a real power system (Bornholm). More technical details can be found in [17]. In this study, the TCLs are assumed to be equipped with such boxes to communicate with the distribution substations. The practical concerns, including the additional costs for the measurement sensors and data privacy, will be investigated in the future work.

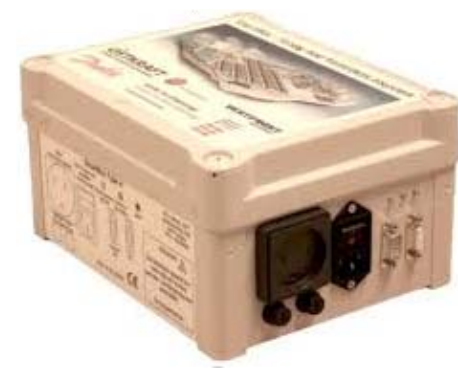

Fig. 4. SmartBox [17]

\section{Algorithms at Distribution Substation Level}

The basic control structure of the distribution substation is shown in Fig. 5. For simplicity, within the same distribution substation, the substation index in the variables is ignored.

\section{A. Estimation of $P_{\mathrm{avi}}^{\mathrm{dn}}$ and $P_{\mathrm{avi}}^{\mathrm{up}}$}

To estimate $P_{\mathrm{avi}}^{\mathrm{dn}}$ and $P_{\mathrm{avi}}^{\mathrm{up}}$, it is essential to find the feasible TCLs which can be fully activated by the trigger frequencies. 


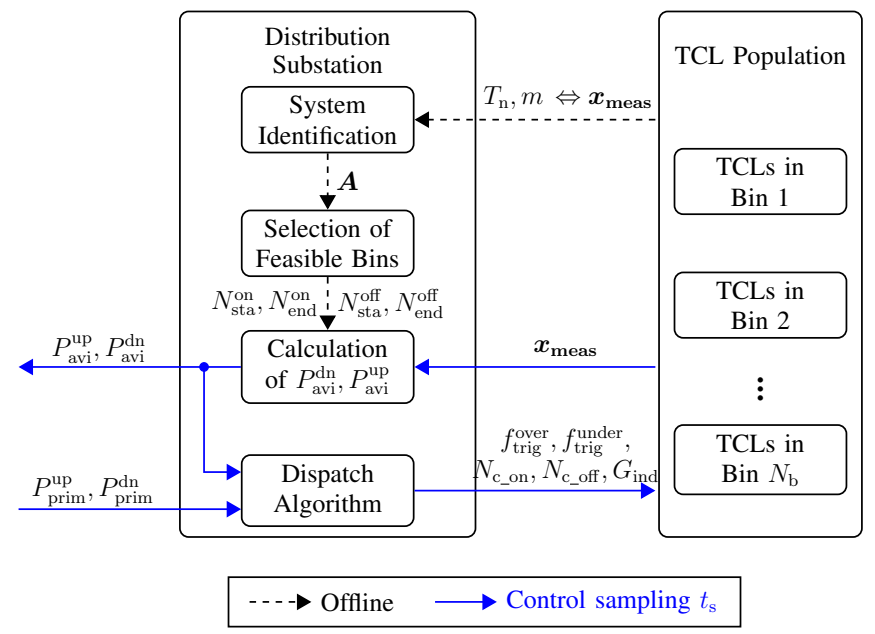

Fig. 5. Basic control structure of the distribution substation

It means that the temperature should be always within the dead-band during the whole control interval. Since $\boldsymbol{A}$ in (3) can estimate the transition probability of TCLs from one bin to the next, the search of feasible TCLs can be changed into the search of feasible bins.

1) Derivation of the A-matrix: According to [12], the matrix $\boldsymbol{A}$ can be analytically calculated with the knowledge of all TCL parameters and ambient temperatures. Alternatively, the matrix $\boldsymbol{A}$ can be identified with full state information (internal normalized temperature $T_{\mathrm{n}}$ and ON/OFF state $m$ ) from all or a subset of the TCL population by using the Hidden Markov Model (HMM) parameter estimation method. If this information is unavailable, a joint parameter and state estimation technique, such as the Extended Kalman Filter (EKF) proposed in [18], might be useful. However, this approach can hardly converge when the number of states becomes large and it is not suitable for online estimation of $\boldsymbol{A}$ [18], [19].

Therefore, in this study, the identification of $\boldsymbol{A}$ is performed offline. The HMM parameter estimation method (Matlab function hmmestimate) is used to identify $\boldsymbol{A}$, which calculates the maximum likelihood estimate of the transition with the sequence of states [20]. Here, $\boldsymbol{x}_{\text {meas }}$ is the full measurement of the state vector $\boldsymbol{x}$. The sampling time for $\boldsymbol{x}_{\text {meas }}$ is set as $t_{\mathrm{m}}=30 \mathrm{~s}$. The last 500 historical points of $\boldsymbol{x}_{\text {meas }}$ are used for the training of $\boldsymbol{A}$, which is updated regularly (1 hour).

2) Selection of feasible bins: Based on the derived $\boldsymbol{A}, \boldsymbol{A}^{k}$ can be calculated, which is $\boldsymbol{A}$ to the $k$ th power. It represents the transition matrix after $k$ steps, expressed by,

$$
\boldsymbol{A}^{k}=\left[\begin{array}{ccc|ccc} 
& \cdots & \cdots & \boldsymbol{A}_{(1, m)}^{k} & \cdots \\
\vdots & \ddots & \vdots & \vdots & \vdots & \vdots \\
\cdots & & \cdots & \boldsymbol{A}_{\left(\frac{N_{\mathrm{b}}}{2}, m\right)}^{k} & \cdots \\
\hline \cdots & \boldsymbol{A}_{\left(\frac{N_{\mathrm{b}}}{2}+1, n\right)}^{k} & \cdots & & \cdots & \\
\vdots & \vdots & \vdots & \vdots & \ddots & \vdots \\
\cdots & \boldsymbol{A}_{\left(N_{\mathrm{b}}, n\right)}^{k} & \cdots & & \cdots &
\end{array}\right]
$$

The matrix element $\boldsymbol{A}_{(i, j)}^{k}$ indicates the transition rate of the $j$ th bin from the last measurement point. By definition of the functions,

$$
\begin{gathered}
f_{\text {on } \rightarrow \text { off }}(n, k)=\alpha_{n}^{k} \triangleq \sum_{i=\frac{N_{\mathrm{b}}}{2}+1}^{N_{\mathrm{b}}} \boldsymbol{A}_{(i, n)}^{k}, \\
f_{\text {off } \rightarrow \text { on }}(m, k)=\beta_{m}^{k} \triangleq \sum_{i=1}^{\frac{N_{\mathrm{b}}}{2}} \boldsymbol{A}_{(i, m)}^{k},
\end{gathered}
$$

$\alpha_{n}^{k}$ indicates the transition rate of the $n$th bin from ON to OFF after $k$ steps.

In this study, the prediction period is equivalent to the control sampling period of TCLs $\left(t_{\mathrm{s}}\right)$, as shown in Fig. 5. Accordingly, the prediction step number $N_{\mathrm{p}}$ is calculated by,

$$
N_{\mathrm{p}}=\frac{t_{\mathrm{s}}}{t_{\mathrm{m}}}
$$

As $k \in\left[1, N_{\mathrm{p}}\right]$, with the increase of $k$, more TCLs transform from $\mathrm{ON}$ to $\mathrm{OFF}$, i.e. $\alpha_{n}^{k}$ becomes larger and reaches its maximum value at $k=N_{\mathrm{p}}$. Similarly, $\beta_{m}^{k}$ indicates the transition rate of the $m$ th bin from OFF to ON after $k$ steps.

Fig. 6 illustrates the feasible bin ranges of ON and OFF devices. For ON bins $\left(\left[1, \frac{N_{\mathrm{b}}}{2}\right]\right)$, the range is $\left[N_{\mathrm{sta}}^{\mathrm{on}}, N_{\mathrm{end}}^{\mathrm{on}}\right]$. $N_{\text {end }}^{\text {on }}$ is set as the ending index. The bins within $\left(N_{\text {end }}^{\text {on }}, \frac{N_{\mathrm{b}}}{2}\right]$ have larger probability to be switched off by the hysteresis temperature control during the control interval. Considering a TCL may be switched off due to the frequency drop, it should not be switched on again by the hysteresis control. Thus, $N_{\mathrm{sta}}^{\mathrm{on}}$ is set as the starting index. If a TCL of the bins within $\left[1, N_{\text {sta }}^{\text {on }}\right)$ is switched off due to the frequency drop, the TCL will change to the neighboring OFF bins within $\left(1+N_{\mathrm{b}}-N_{\mathrm{sta}}^{\mathrm{on}}, N_{\mathrm{b}}\right]$, which have larger probability to be switched on by the hysteresis temperature control.

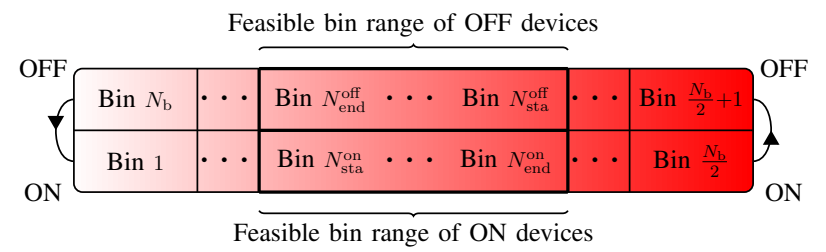

Fig. 6. Feasible bin ranges of ON/OFF bins

For OFF bins $\left(\left[\frac{N_{\mathrm{b}}}{2}+1, N_{\mathrm{b}}\right]\right)$, the range is $\left[N_{\mathrm{sta}}^{\text {off }}, N_{\text {end }}^{\text {off }}\right]$. $N_{\text {end }}^{\text {off }}$ is set as the ending index. The bins within $\left(N_{\text {end }}^{\text {off }}, N_{\mathrm{b}}\right]$ have larger probability to be switched on by the hysteresis temperature control during the control interval. Obviously, ( $\left.N_{\text {end }}^{\text {off }}, N_{\mathrm{b}}\right]$ is equivalent to $\left(1+N_{\mathrm{b}}-N_{\text {sta }}^{\text {on }}, N_{\mathrm{b}}\right]$. Therefore,

$$
N_{\text {end }}^{\text {off }}=1+N_{\mathrm{b}}-N_{\text {sta }}^{\text {on }} \Rightarrow N_{\text {sta }}^{\text {on }}=1+N_{\mathrm{b}}-N_{\text {end }}^{\text {off }} .
$$

Considering a TCL may be switched on due to the frequency drop, it should not be switched off again by the hysteresis control. Thus, $N_{\mathrm{sta}}^{\text {off }}$ is set as the starting index. If a TCL of the bins within $\left[\frac{N_{\mathrm{b}}}{2}+1, N_{\mathrm{sta}}^{\mathrm{off}}\right)$ is switched on due to the frequency rise, the TCL will change to the neighboring $\mathrm{ON}$ bins within $\left(1+N_{\mathrm{b}}-N_{\mathrm{sta}}^{\text {off }}, \frac{N_{\mathrm{b}}}{2}\right]$, which have larger probability to be switched off by the hysteresis temperature control. Obviously, $\left(1+N_{\mathrm{b}}-N_{\mathrm{sta}}^{\text {off }}, \frac{N_{\mathrm{b}}}{2}\right]$ is equivalent to $\left(N_{\mathrm{end}}^{\mathrm{on}}, \frac{N_{\mathrm{b}}}{2}\right]$. Therefore,

$$
1+N_{\mathrm{b}}-N_{\mathrm{sta}}^{\mathrm{off}}=N_{\mathrm{end}}^{\mathrm{on}} \Rightarrow N_{\mathrm{sta}}^{\mathrm{off}}=1+N_{\mathrm{b}}-N_{\mathrm{end}}^{\mathrm{on}} .
$$

Therefore, only $N_{\text {end }}^{\text {on }}$ and $N_{\text {end }}^{\text {off }}$ are required.

Two methods are proposed to decide $N_{\text {end }}^{\text {on }}$ and $N_{\text {end }}^{\text {off }}$. 
a) Method I: This method only considers the transition rate of bins at the last prediction step, i.e. $k=N_{\mathrm{p}}$. The feasible bins are those whose transition rates between ON and OFF are small, i.e. these bins wound not transit between $\mathrm{ON}$ and $\mathrm{OFF}$ without an external input. $N_{\text {end }}^{\text {on }}$ can be regarded as the critical point,

$$
\begin{aligned}
& f_{\text {on } \rightarrow \text { off }}\left(n, N_{\mathrm{p}}\right) \leq \alpha_{\text {set }}, \forall n \in\left[1, N_{\text {end }}^{\text {on }}\right], \\
& f_{\text {on } \rightarrow \text { off }}\left(n, N_{\mathrm{p}}\right)>\alpha_{\text {set }}, \forall n \in\left(N_{\text {end }}^{\text {on }}, \frac{N_{\mathrm{b}}}{2}\right],
\end{aligned}
$$

where $\alpha_{\text {set }}$ is the predefined threshold of the transition rate from ON to OFF. In this study, $\alpha_{\text {set }}=10 \%$. In the same way, $N_{\text {end }}^{\text {off }}$ can be determined by,

$$
\begin{aligned}
& f_{\text {off } \rightarrow \text { on }}\left(n, N_{\mathrm{p}}\right) \leq \beta_{\text {set }}, \forall n \in\left[\frac{N_{\mathrm{b}}}{2}+1, N_{\text {end }}^{\text {off }}\right], \\
& f_{\text {on } \rightarrow \text { off }}\left(n, N_{\mathrm{p}}\right)>\beta_{\text {set }}, \forall n \in\left(N_{\text {end }}^{\text {off }}, N_{\mathrm{b}}\right],
\end{aligned}
$$

where $\beta_{\text {set }}$ is the predefined threshold of the transition rate from OFF to ON. In this study, $\beta_{\text {set }}=10 \%$.

b) Method II: This method considers the transition rate of bins in the whole control interval, $N_{\text {end }}^{\text {on }}$ can be derived by,

$$
\begin{aligned}
& \sum_{k=1}^{N_{\mathrm{p}}} f_{\text {on } \rightarrow \text { off }}(n, k) \leq \alpha_{\text {set }}, \forall n \in\left[1, N_{\text {end }}^{\text {on }}\right], \\
& \sum_{k=1}^{N_{\mathrm{p}}} f_{\text {on } \rightarrow \text { off }}(n, k)>\alpha_{\text {set }}, \forall n \in\left(N_{\text {end }}^{\text {on }}, \frac{N_{\mathrm{b}}}{2}\right],
\end{aligned}
$$

$N_{\text {end }}^{\text {off }}$ can be derived by,

$$
\begin{aligned}
& \sum_{k=1}^{N_{\mathrm{p}}} f_{\text {off } \rightarrow \text { on }}(n, k) \leq \beta_{\text {set }}, \forall n \in\left[\frac{N_{\mathrm{b}}}{2}+1, N_{\text {end }}^{\text {off }}\right], \\
& \sum_{k=1}^{N_{\mathrm{p}}} f_{\text {on } \rightarrow \text { off }}(n, k)>\beta_{\text {set }}, \forall n \in\left(N_{\text {end }}^{\text {off }}, N_{\mathrm{b}}\right] .
\end{aligned}
$$

Comparably, the result of Method II is more conservative, in other words, the feasible bin range derived by Method II is narrower than that derived by Method I. In this study, Method $\mathrm{I}$ is adopted for the simulations.

\section{B. Calculation of $P_{\mathrm{avi}}^{\mathrm{dn}}$ and $P_{\mathrm{avi}}^{\mathrm{up}}$}

Accordingly, $P_{\text {avi }}^{\mathrm{dn}}$ and $P_{\mathrm{avi}}^{\mathrm{up}}$ can be calculated by,

$$
\begin{aligned}
& P_{\text {avi }}^{\mathrm{dn}}=\sum_{n=N_{\mathrm{sta}}^{\text {on }}}^{N_{\text {end }}^{\text {on }}} \boldsymbol{x}_{\text {meas }}(n) P_{\mathrm{sum}}, \\
& P_{\text {avi }}^{\text {up }}=\sum_{n=N_{\mathrm{sta}}^{\text {off }}}^{N_{\text {end }}^{\text {off }}} \boldsymbol{x}_{\text {meas }}(n) P_{\mathrm{sum}},
\end{aligned}
$$

where $\boldsymbol{x}_{\text {meas }}(n)$ is the $n$th element of the state vector measurement $\boldsymbol{x}_{\text {meas }}$, which represents the fraction of TCLs in the $n$th bin, and $P_{\text {sum }}$ is the power summation of all TCLs governed by the same substation.

It should be noticed that if the TCLs are not triggered by the frequency rise or fall during the frequency event (overor under-frequency), $\boldsymbol{u}$ in (3) can be ignored. Otherwise, the TCLs will be triggered and $\boldsymbol{u}$ can't be ignored. Accordingly, the state vector $\boldsymbol{x}$ in (3) will be affected. However, the state vector measurement $\boldsymbol{x}_{\text {meas }}$ will be updated at the next control point. Based on the updated $\boldsymbol{x}_{\text {meas }}$, the calculation of $P_{\text {avi }}^{\text {up }}$ and $P_{\mathrm{avi}}^{\mathrm{dn}}$ for the next control period $t_{\mathrm{s}}$ will be redone. Thus, the effect on the bins during the last control period won't affect the control for the next control period.
Theoretically, more bins can lead to a more precise estimation of $P_{\mathrm{avi}}^{\mathrm{dn}}$ and $P_{\mathrm{avi}}^{\mathrm{up}}$. Due to the constraints of the measurement accuracy, the total bin number $N_{\mathrm{b}}$ is limited. In this study, the temperature measurement accuracy is $0.1^{\circ} \mathrm{C}$ and $N_{\mathrm{b}}=40$.

\section{State estimation with Kalman filter}

Although the state measurement $\boldsymbol{x}_{\text {meas }}$ is available, it is beneficial to use a Kalman filter, because the TCL aggregated model (3) is imperfect. As concluded by [6], instead of the perfect state measurements, using an imperfect model together with imperfect state estimates can result in better control performance. Accordingly, the basic control structure in Fig. 5 can be modified to the one in Fig. 7, which is adopted for the simulations.

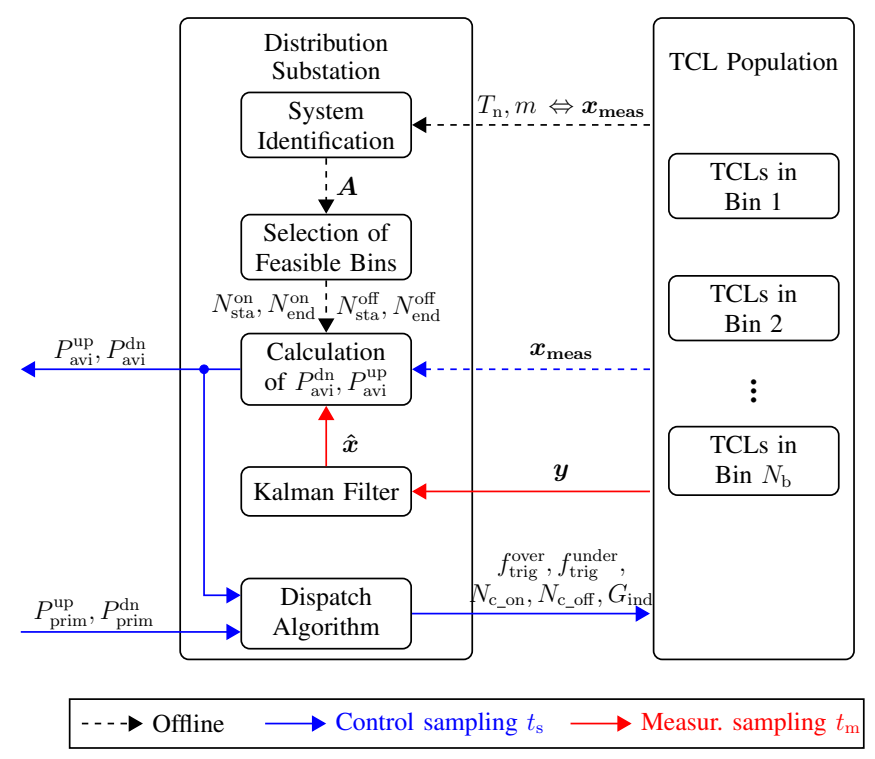

Fig. 7. Modified control structure with Kalman filter

A standard Kalman filter is designed for the state estimation based on the following system,

$$
\begin{aligned}
\boldsymbol{x}(k+1) & =\boldsymbol{A} \boldsymbol{x}(k)+\boldsymbol{B} \boldsymbol{u}(k)+\boldsymbol{B}_{\boldsymbol{w}} \boldsymbol{w}(k), \\
\boldsymbol{y}(k) & =\boldsymbol{C} \boldsymbol{x}(k)+\boldsymbol{v}(k),
\end{aligned}
$$

where $\boldsymbol{w} \in \mathbb{R}^{N_{\mathrm{b}} \times 1}$ and $\boldsymbol{v} \in \mathbb{R}^{1 \times 1}$ indicate the process and measurement noise, respectively. They are assumed to be independent, white, and with normal probability distributions. The covariance matrix of $\boldsymbol{w}$ is defined by $\boldsymbol{Q}_{\mathbf{k f}} \in \mathbb{R}^{N_{\mathrm{b}} \times N_{\mathrm{b}}}$ and the covariance of $\boldsymbol{v}$ is defined by $\boldsymbol{R}_{\mathbf{k f}} \in \mathbb{R}^{1 \times 1}$. The time and measurement updates are described by (18)-(22),

\section{Time update equations:}

$$
\begin{aligned}
& \hat{\boldsymbol{x}}^{-}(k)=\boldsymbol{A} \hat{x}(k-1)+\boldsymbol{B} \boldsymbol{u}(k), \\
& \boldsymbol{P}^{-}(k)=\boldsymbol{A} \boldsymbol{P}(k-1) \boldsymbol{A}^{\prime}+\boldsymbol{Q}_{\mathbf{k f}},
\end{aligned}
$$

Measurement update equations:

$$
\begin{aligned}
\boldsymbol{K}(k) & =\boldsymbol{P}^{-}(k) \boldsymbol{C}^{\prime}\left(\boldsymbol{C} \boldsymbol{P}^{-}(k) \boldsymbol{C}^{\prime}+\boldsymbol{R}_{\mathbf{k f}}\right)^{-1}, \\
\hat{\boldsymbol{x}}(k) & =\hat{\boldsymbol{x}}^{-}(k)+\boldsymbol{K}(k)\left(\boldsymbol{y}(k)-\boldsymbol{C} \hat{\boldsymbol{x}}^{-}(k)\right), \\
\boldsymbol{P}(k) & =(\boldsymbol{I}-\boldsymbol{K}(k) \boldsymbol{C}) \boldsymbol{P}^{-}(k),
\end{aligned}
$$


where $\hat{\boldsymbol{x}}^{-}$and $\hat{\boldsymbol{x}}$ are the predicted (priori) and updated (posteriori) state estimate, respectively, $\boldsymbol{K}$ is the optimal Kalman gain, and $\boldsymbol{P}^{-}$and $\boldsymbol{P}$ are the predicted (priori) and updated (posteriori) state estimate covariance, respectively.

Accordingly, the calculation of $P_{\mathrm{avi}}^{\mathrm{dn}}$ and $P_{\mathrm{avi}}^{\mathrm{up}}$ in (16) will be changed to,

$$
\begin{aligned}
& P_{\mathrm{avi}}^{\mathrm{dn}}=\sum_{n=N_{\mathrm{sta}}^{\mathrm{on}}}^{N_{\mathrm{end}}^{\mathrm{on}}} \hat{\boldsymbol{x}}(n) P_{\mathrm{sum}}, \\
& P_{\mathrm{avi}}^{\mathrm{up}}=\sum_{n=N_{\mathrm{sta}}^{\text {off }}}^{N_{\text {end }}^{\text {uff }}} \hat{\boldsymbol{x}}(n) P_{\mathrm{sum}} .
\end{aligned}
$$

\section{Dispatch of control signals for TCLs}

The TCLs within the feasible bins are considered not to be affected by the local hysteresis control and reliable for the potential frequency response during the next control interval. The merit order method is used to dispatch the control signals. The derived feasible TCLs are ranked for switching according to the predefined criterion, such as the bid price and temperature. For the latter case, TCLs in bins closer to the edge of the dead-band have a higher ranking in the list, as they are about to "naturally" switch and therefore the total number of switching times is minimized.

Following the derived merit order, the feasible TCLs are divided into several groups according to $P_{\text {avi }}^{\text {dn }}, P_{\text {avi }}^{\text {up }}, P_{\text {prim }}^{\text {dn }}$ and $P_{\text {prim. }}^{\text {up }}$ Specifically,

$$
N_{\text {c_on }}=\left\lfloor\frac{P_{\text {avi }}^{\text {dn }}}{P_{\text {prim }}^{\text {dn }}}\right\rfloor, N_{\text {c_off }}=\left\lfloor\frac{P_{\text {avi }}^{\text {up }}}{P_{\text {prim }}^{\text {up }}}\right\rfloor,
$$

where $N_{\text {c_on }_{-}}$and $N_{\text {c_off }_{-}}$are the group numbers for $\mathrm{ON}$ and OFF devices, respectively. The total power of each group corresponds to the reserve requirement of over and under frequency events, as illustrated in Fig. 8.

Conventionally, ON TCLs are only required to respond to the under-frequency event, i.e. the dispatched trigger frequency $f_{\text {trig }}^{\text {under }} \in\left[f_{\text {min }}, f_{\text {ref }}-\Delta f_{\mathrm{db}}\right]$. They are switched off when

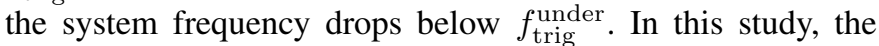
operation of ON TCLs is extended to the over-frequency event and the over-frequency trigger $f_{\text {trig }}^{\text {over }}$ is also dispatched, similar to the case of OFF TCLs. Moreover, the dispatched control signals also include the group index $G_{\text {ind }}, N_{\text {c_on }}$ and $N_{\text {c_off }}$. This control settings can guarantee the control performance with reduced short-cycling probability of devices. More details are described in Section VI.

According to the merit order, the corresponding trigger frequencies ( $f_{\text {trig }}^{\text {over }}, f_{\text {trig }}^{\text {under }}$ ) and group index $\left(G_{\text {ind }}\right)$ of a TCL can be determined. Suppose the merit order is $K_{\text {ord }}$,

$$
p=\left\lfloor\frac{\sum_{i=1}^{K_{\text {ord }}} P_{\text {rate } \_i}}{P_{\text {prim }}^{\text {dn }}}\right\rfloor, q=\frac{\sum_{i=1}^{K_{\text {ord }}} P_{\text {rate } \_} i}{P_{\text {prim }}^{\text {dn }}}-p,
$$

For ON devices,

$$
\begin{aligned}
G_{\text {ind }} & =p+1, \\
f_{\text {trig }}^{\text {under }} & =f_{\text {ref }}-\Delta f_{\text {db }}-q\left(f_{\text {ref }}-\Delta f_{\text {db }}-f_{\text {min }}\right), \\
f_{\text {trig }}^{\text {over }} & =2 f_{\text {ref }}-f_{\text {trig }}^{\text {under }} .
\end{aligned}
$$

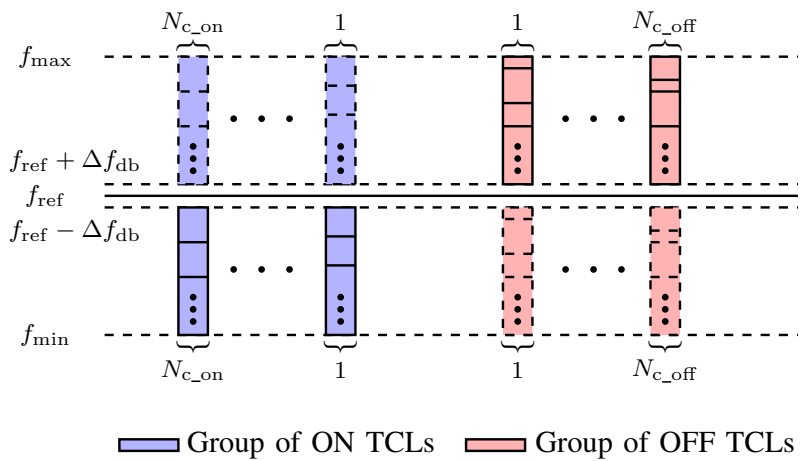

Fig. 8. Grouping of ON/OFF TCLs

For OFF devices,

$$
\begin{aligned}
G_{\text {ind }} & =p+1, \\
f_{\text {trig }}^{\text {over }} & =f_{\text {ref }}+\Delta f_{\text {db }}+q\left(f_{\text {max }}-f_{\text {ref }}-\Delta f_{\mathrm{db}}\right), \\
f_{\text {trig }}^{\text {under }} & =2 f_{\text {ref }}-f_{\text {trig }}^{\text {over }} .
\end{aligned}
$$

\section{LOCAL CONTROLlER DESIGN FOR TCLS}

\section{A. Working principle}

In this section, the working principle of the proposed local controller is explained. With the derived group numbers $\left(N_{\text {c_on }}, N_{\text {c_off }}\right)$, the group index $\left(G_{\text {ind }}\right)$, and the trigger frequencies ( $f_{\text {trig }}^{\text {over }}, f_{\text {trig }}^{\text {under }}$ ), the local controller makes the TCLs autonomously respond to the frequency variation in sequence and the aggregated power fulfills the requirement of primary frequency control. Especially for the case $N_{\text {c_一o }_{1}}>1$ or $N_{\text {c_off }}>1$, when the frequency oscillates during a short period, the TCLs of different groups take turns at responding to the frequency, which can significantly avoid the short-cycling of individual TCLs.

A typical under-frequency event with oscillation during a control interval is shown in Fig. 9. It is similar for the overfrequency event $\left(\left|f_{\max }-f_{\text {ref }}\right|=\left|f_{\max }-f_{\text {ref }}\right|\right)$.

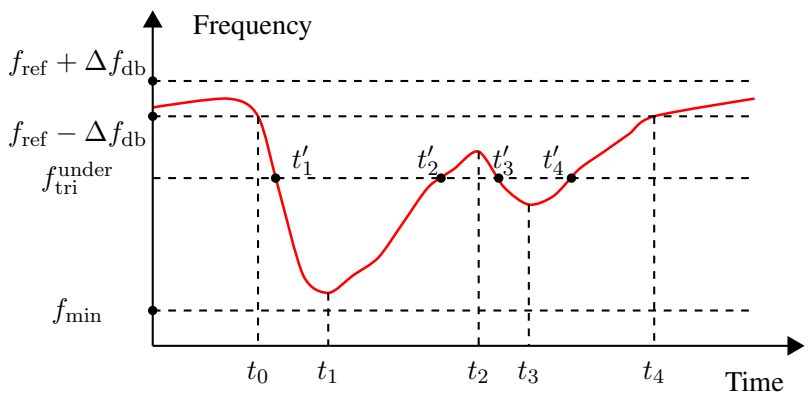

Fig. 9. Frequency variation

According to the variation trend, the whole process can be divided into 4 phases. Suppose $N_{\text {c_on }}=N_{\text {c_off }}=2$. G1 and $\mathrm{G} 2$ are the ON TCLs with the same trigger frequency $f_{\text {trig }}^{\text {under }}$ and their group indices are $G_{\text {ind }}=1$ and $G_{\text {ind }}=2$, respectively. G3 and G4 are the OFF TCLs with the same trigger frequency $f_{\text {trig }}^{\text {over }}$ and their group indices are $G_{\text {ind }}=1$ and $G_{\text {ind }}=2$, respectively. The switching activities are shown in Fig. 10. 


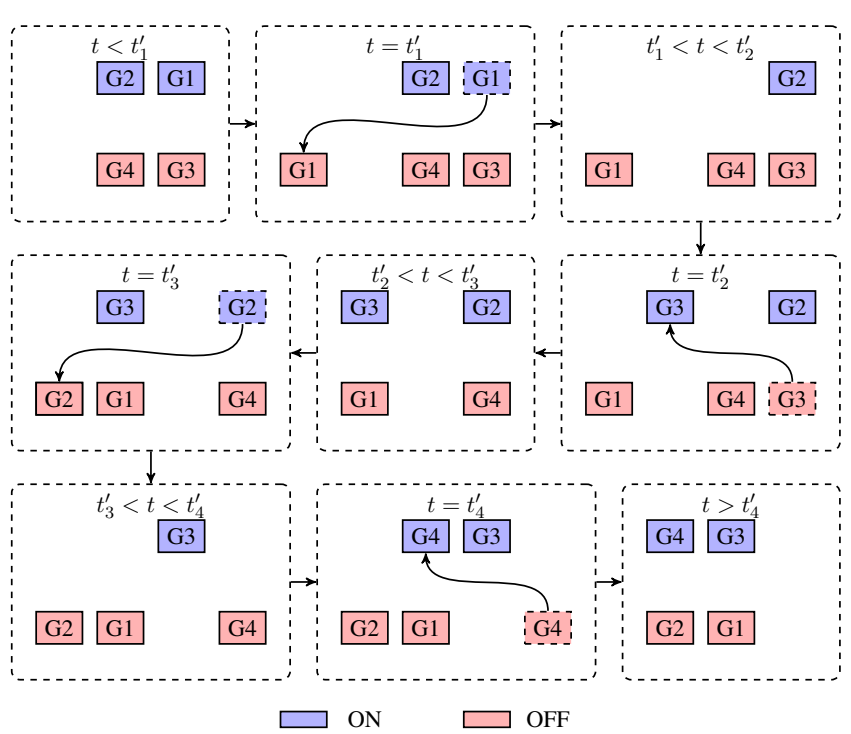

Fig. 10. Switching activities during the under frequency event

Phase 1: $t_{0} \sim t_{1}$. At $t_{1}^{\prime}$, the frequency drops below $f_{\text {trig }}^{\text {under }}$ and G1 is firstly activated to respond to the frequency drop. It is switched from ON to OFF. Accordingly, G1 is removed from the ON queue and pushed into the OFF queue. G2 moves forward and becomes the first entry of the ON queue.

Phase 2: $t_{1} \sim t_{2}$. The frequency starts increasing and goes above $f_{\text {trig }}^{\text {under }}$ at $t_{2}^{\prime}$. As the first entry of the OFF queue, G3 is switched from OFF to ON. Accordingly, G3 is removed from the OFF queue and pushed into the ON queue. G4 moves forward and becomes the first entry of the OFF queue.

Phase 3: $t_{2} \sim t_{3}$. The frequency drops again and goes below $f_{\text {trig }}^{\text {under }}$ at $t_{3}^{\prime}$. As the first entry of the ON queue, G2 is switched from ON to OFF. Accordingly, G2 is removed from the ON queue and pushed into the OFF queue. G4 moves forward in the OFF queue.

Phase 4: $t_{3} \sim t_{4}$. The frequency recovers and goes above $f_{\text {trig }}^{\text {under }}$ at $t_{4}^{\prime}$. As the first entry of the OFF queue, G4 is switched from OFF to ON. Accordingly, G4 is removed from the OFF queue and pushed into the ON queue.

Without the grouping and participation of the OFF TCLs, the switching of G1 would be very frequent, i.e. 4 times in this scenario. With the proposed algorithm, the switching activities are distributed among different TCLs. Therefore, the short cycling number is largely reduced.

\section{B. Implementation}

The configuration of the proposed local controller is shown in Fig. 11. The scheme starts with the frequency measurement at a time step, e.g. $0.02 \mathrm{~s}$. A first order filter with time constant, e.g. $0.5 \mathrm{~s}$, is used to reduce measurement noise. The difference between the measured frequency $f_{\mathrm{m}}$ and received trigger frequencies $\left(f_{\mathrm{trig}}^{\text {over }}\right.$ and $\left.f_{\mathrm{trig}}^{\text {under }}\right)$ are delivered to the edge detector to examine the rising or falling edges. The output
Ctr1 is expressed by,

$$
\begin{aligned}
& \operatorname{Ctr} 1(t)= \\
& \left\{\begin{array}{cc}
1 & \text { if }\left(\Delta f_{\text {over }} \text { rises across } 0 \wedge m(t-\Delta t)=0\right) \vee \\
-1 & \begin{array}{l}
\text { if }\left(\Delta f_{\text {under }} \text { rises across } 0 \wedge m(t-\Delta t)=0\right), \\
\text { over falls across } 0 \wedge m(t-\Delta t)=1) \vee
\end{array} \\
0 & \left(\Delta f_{\text {under }} \text { falls across } 0 \wedge m(t-\Delta t)=1\right), \\
\text { otherwise. }
\end{array}\right.
\end{aligned}
$$

where $\Delta f_{\text {over }} \triangleq f_{\mathrm{m}}-f_{\text {trig }}^{\text {over }}$ and $\Delta f_{\text {under }} \triangleq f_{\mathrm{m}}-f_{\text {trig }}^{\text {under }}$. Ctr1 is then used as the selection criterion in Selector 1 to decide the switching command $S_{1}$. The control logic of $S_{1}$ is,

$$
S_{1}(t)= \begin{cases}1 & \text { if } \operatorname{Ctr} 1=1, \\ 0 & \text { if Ctr1 }=-1, \\ m(t-\Delta t) & \text { otherwise, }\end{cases}
$$

where $\mathrm{ON}$ is 1 and OFF is 0 .

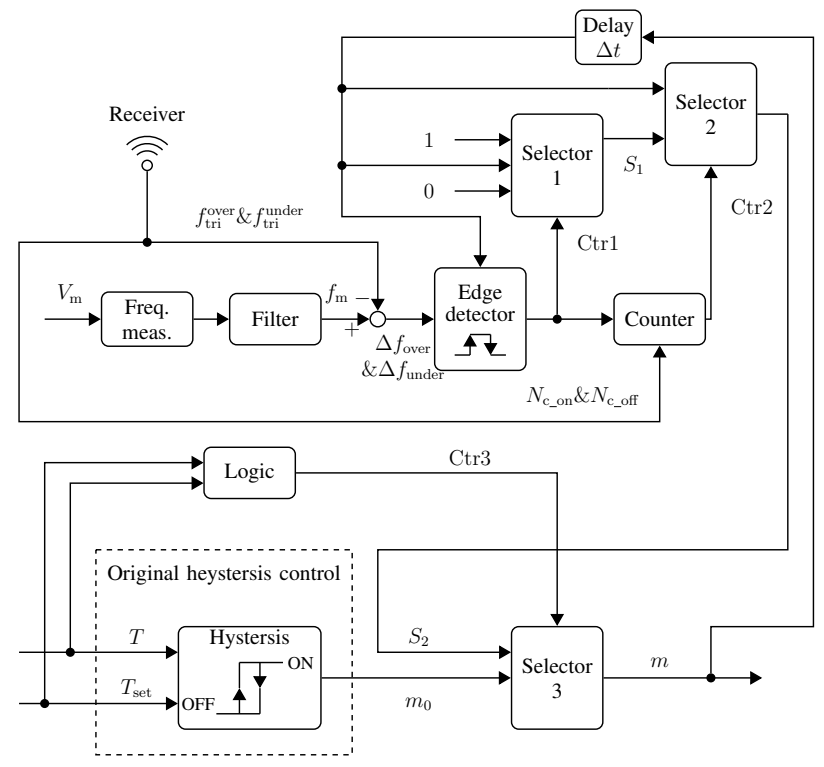

Fig. 11. Local controller of TCL

The control signal Ctr2 is used to indicate the activation sequence in the queue, described in Section VI-A. It can be considered as a counter. The logic is as follows,

$\operatorname{Ctr} 2(t)=$

$\begin{cases}\operatorname{Ctr} 2(t-\Delta t)-1 & \text { if } \operatorname{Ctr} 2(t-\Delta t) \geq 1 \wedge \operatorname{Ctr} 1(t) \neq 0, \\ N_{\mathrm{c} \_o n} & \text { if } \operatorname{Ctr} 2(t-\Delta t)=0 \wedge \operatorname{Ctr} 1(t)=1, \\ N_{\text {c_off }} & \text { if } \operatorname{Ctr} 2(t-\Delta t)=0 \wedge \operatorname{Ctr} 1(t)=-1, \\ \operatorname{Ctr} 2(t-\Delta t) & \text { Otherwise. }\end{cases}$

The switching command $S_{2}$ can be decided by,

$$
S_{2}(t)= \begin{cases}S_{1}(t) & \text { if Ctr2 }=0, \\ m(t-\Delta t) & \text { otherwise. }\end{cases}
$$

As the output of the frequency control loop, $S_{2}$ is then merged into the original hysteresis control to determine the final switching command $m$ through Selector 3. By detecting if the TCL is within its temperature dead-band,

$$
\operatorname{Ctr} 3(t)= \begin{cases}1 & \text { if } T_{\text {set }}-\frac{\delta_{\mathrm{db}}}{2} \leq T(t) \leq T_{\mathrm{set}}+\frac{\delta_{\mathrm{db}}}{2}, \\ 0 & \text { otherwise. }\end{cases}
$$


The selection logic of Selector 3 is,

$$
m(t)= \begin{cases}S_{2}(t) & \text { if Ctr3 }=1 \\ m_{0}(t) & \text { otherwise. }\end{cases}
$$

Obviously, compared with the proposed local control loop, the existing hysteresis control has a higher priority. In other words, the TCL device is triggered to respond to the frequency event only when the temperature is within its dead-band $\delta_{\mathrm{db}}$. Since $\delta_{\mathrm{db}}$ is small $\left(2^{\circ} \mathrm{C}\right.$ in this study), such a small change around the temperature reference will not affect the customer comfort level.

The measured frequency will decide whether and when the TCLs will be triggered. Therefore, the accuracy of the frequency measurement device applied in the local control loop in Fig. 11 can have a significant impact on the control performance. To what extent the control performance is affected will be discussed in the simulations in Section VII.

\section{Simulation Results}

The power system of Bornholm, a Danish island in the Baltic Sea, is used as the test system for the simulation. As shown in Fig. 12, the power system comprises a ringshaped transmission grid of $60 \mathrm{kV}$. Most households have heat pumps and electric heatings. When the system operates in the islanding mode, the frequency stability becomes problematic, which make it necessary for TCLs to provide frequency support.

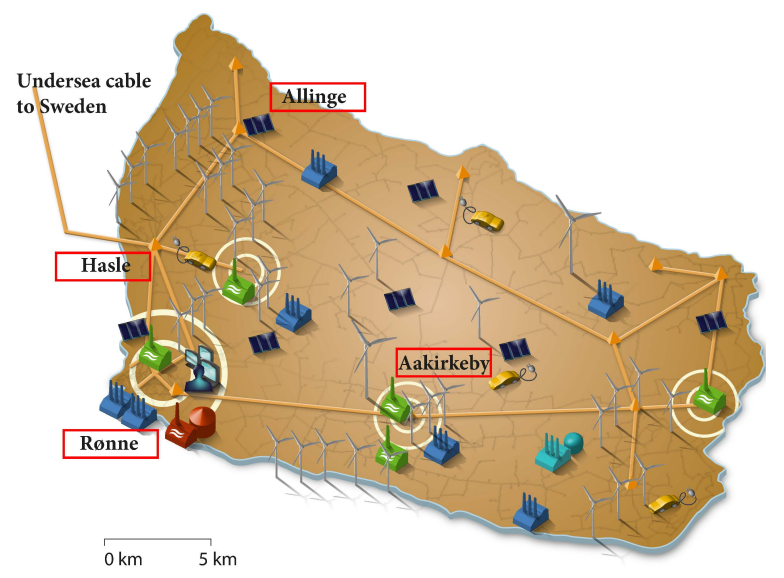

Fig. 12. Bornholm power system

In this study, 1000 TCLs are integrated into the grid and divided into 4 groups (250 TCLs each). Accordingly, these groups are managed by 4 distribution substations-Hasle, Rnne, Aakirkeby and Allinge, labelled in Fig. 12. The parameters of TCLs are listed in Table II.

$P_{\text {rate }}$ and COP are derived from the installed Bosch EHP AA 6.5 Air Source Heat Pump (ASHP), according to [21]. Due to the variety of different customers, $P_{\text {rate }}$ is assumed uniformly distributed between $6 \mathrm{~kW}$ and $8 \mathrm{~kW}$. Based on the assumption that the average living area is $250 \mathrm{~m}^{2}$, the average value of the thermal capacitance $C$ is assumed to be $0.04 \mathrm{kWh} /{ }^{\circ} \mathrm{C} \times 250 \mathrm{~m}^{2}=10 \mathrm{kWh} /{ }^{\circ} \mathrm{C}$. The average value of the thermal resistance $R$ is assumed to be $\left(0.002 \mathrm{~kW} /{ }^{\circ} \mathrm{C} / \mathrm{m}^{2} \times 250 \mathrm{~m}^{2}\right)^{-1}=2{ }^{\circ} \mathrm{C} / \mathrm{kW} . R$ and $C$ are
TABLE II

TCL PARAMETERS

\begin{tabular}{l|l|l}
\hline \hline Parameter & Value range & Distribution \\
\hline \hline$T_{\text {set }}$ & $15 \sim 20^{\circ} \mathrm{C}$ & Uniform distribution \\
$T_{\mathrm{a}}$ & $5^{\circ} \mathrm{C}$ & - \\
$C$ & $8 \sim 12 \mathrm{kWh} /{ }^{\circ} \mathrm{C}$ & Uniform distribution \\
$R$ & $1.5 \sim 2.5^{\circ} \mathrm{C} / \mathrm{kW}$ & Uniform distribution \\
$\delta_{\mathrm{db}}$ & $2^{\circ} \mathrm{C}$ & - \\
$P_{\text {rate }}$ & $6 \sim 8 \mathrm{~kW}$ & Uniform distribution \\
$\mathrm{COP}$ & 3 & - \\
\hline
\end{tabular}

also assumed to be uniformly distributed, which are common statistic values from [22].

\section{A. Estimation of aggregated model of TCLs}

According to the derived $\boldsymbol{A}$, three scenarios are defined with different control sampling time $t_{\mathrm{s}}$, as listed in Table III. The forecast horizon is equal to $t_{\mathrm{s}}$.

TABLE III

SCENARIO DEFINITION

\begin{tabular}{c|c}
\hline \hline Scenario & Definition \\
\hline \hline Ind & Individual TCL model \\
Agg01 & Aggregated model with $t_{\mathrm{s}}=1 \mathrm{~min}$ \\
Agg02 & Aggregated model with $t_{\mathrm{s}}=2 \mathrm{~min}$ \\
Agg05 & Aggregated model with $t_{\mathrm{s}}=5 \mathrm{~min}$ \\
\hline
\end{tabular}

The estimated aggregated power of different scenarios, equivalent to $\boldsymbol{y}$ in (3), are simulated and compared with that of the individual TCL model to evaluate the estimation accuracy. The simulation period is 3600 seconds. The simulation results are shown in Fig. 13.

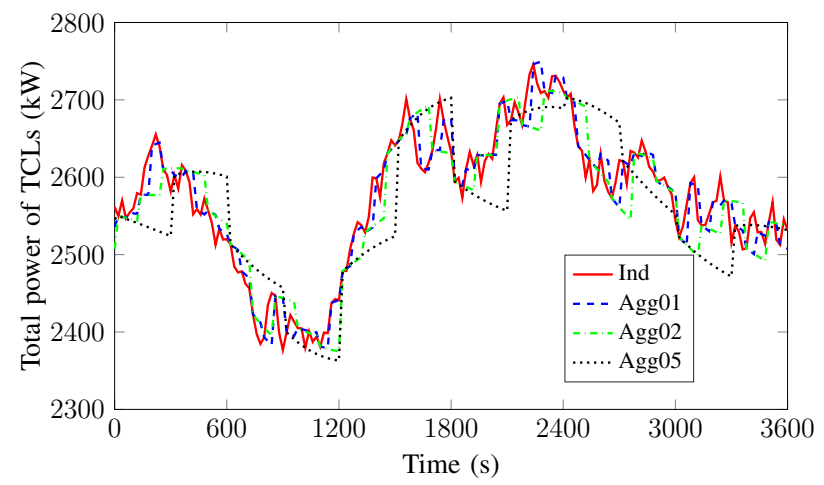

Fig. 13. Comparison of aggregated power of TCLs

To quantify the estimation error between the aggregated model and the individual TCL model, the Root Mean Square Errors (RMSEs) of the aggregated power of different scenarios are calculated and listed in Table IV. It can be observed that the power of the model with a smaller control period can track the power of the individual TCL model more precisely, which implies better estimation capability. However, a smaller $t_{\mathrm{s}}$ leads to a higher requirement of the communication infrastructure. 
To strike a balance, $t_{\mathrm{s}}=2 \mathrm{~min}$ is adopted in this study as its RMSE is only $32.3 \mathrm{~kW}$, which is quite small compared to the total TCL power capacity.

TABLE IV

RMSE FOR DIFFERENT SCENARIOS

\begin{tabular}{c|c}
\hline \hline Scenario & RMSE \\
\hline \hline Agg01 & $23.3 \mathrm{~kW}$ \\
Agg02 & $32.3 \mathrm{~kW}$ \\
Agg05 & $45.9 \mathrm{~kW}$ \\
\hline
\end{tabular}

\section{B. Frequency response}

In this subsection, the proposed controller is tested under both over- and under-frequency events. Two factors, which have impacts on the control performance, are taken into account. Firstly, the accuracy of the frequency measurement device, applied in the local control loop in Fig. 11, is considered. Three accuracy levels are defined, including $\pm 0.001 \mathrm{~Hz}$ (Acc01), $\pm 0.005 \mathrm{~Hz}$ (Acc05) and $\pm 0.01 \mathrm{~Hz}$ (Acc10). Secondly, the frequency responses at different time points within $t_{\mathrm{s}}$ are tested to evaluate whether the proposed controller can cover the whole interval. Since $t_{\mathrm{s}}=2 \mathrm{~min}$, the time of the frequency event $t_{\text {event }}$ is set to $5 \mathrm{~s}, 55 \mathrm{~s}$ and $105 \mathrm{~s}$, respectively. In this simulation, the estimated power reserve references for up and down regulation are derived based on (9)-(23): $P_{\mathrm{avi}}^{\mathrm{up}}=2120 \mathrm{~kW}, P_{\mathrm{avi}}^{\mathrm{dn}}=2260 \mathrm{~kW}$. The references for power reserves are $P_{\text {prim }}^{\text {up }}=P_{\text {prim }}^{\text {dn }}=1000 \mathrm{~kW}$.

1) Under-frequency event: Fig. 14 shows the frequency response at $t_{\text {event }}=55 \mathrm{~s}$. The frequency drops due to a sudden generation loss. It can be observed that compared to the case without TCLs, the frequency response with TCLs can efficiently increase the frequency nadir and shorten the recovery time (Fig. 14(a)). The probability density curves are used to compare the frequency responses of TCLs with different measurement accuracy levels (Fig. 14(b)) and no big differences are found among them.

The comparison of aggregated power responses of TCLs $P_{\mathrm{tcl}}^{\mathrm{res}}$ is shown in Fig. 15. It can be observed that the TCLs with higher measurement accuracy can track the frequency variation more smoothly (Fig. 15(a)). $\Delta P_{\mathrm{tcl}}^{\mathrm{dev}}$, defined by,

$$
\Delta P_{\mathrm{tcl}}^{\mathrm{dev}}(t)=P_{\mathrm{tcl}}^{\mathrm{res}}(t)-\left(f(t)-\Delta f_{\mathrm{db}}\right) \frac{P_{\mathrm{prim}}^{\mathrm{dn}}}{f_{\min }-\Delta f_{\mathrm{db}}},
$$

represents the control error. As shown in Fig. 15(b), the TCLs with higher measurement accuracy shows better control performance.

The RMSEs of $\Delta P_{\mathrm{tcl}}^{\mathrm{dev}}$ are used to quantify the control performances, as listed in Table V. With the same measurement accuracy, the differences of RMSEs at different event time points are quite small, which verifies the proposed control throughout the whole control period. Besides, a higher accuracy level leads to a smaller RMSE value, which implies a better tracking performance.

The switching statistics for the under-frequency event are listed in Table VI. Here, $n_{\text {switch }}$ indicates the total switching number, which considers only the switching activities for the (a)

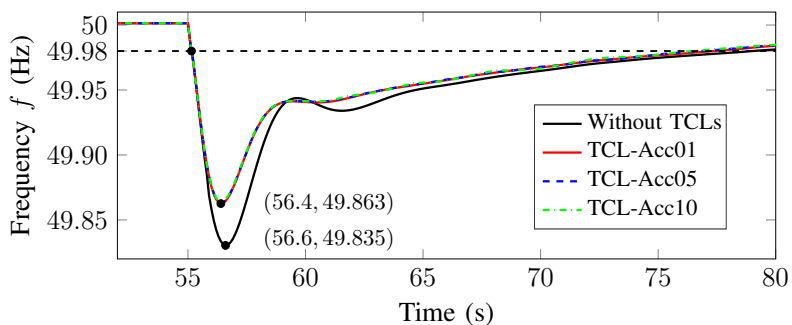

(b)

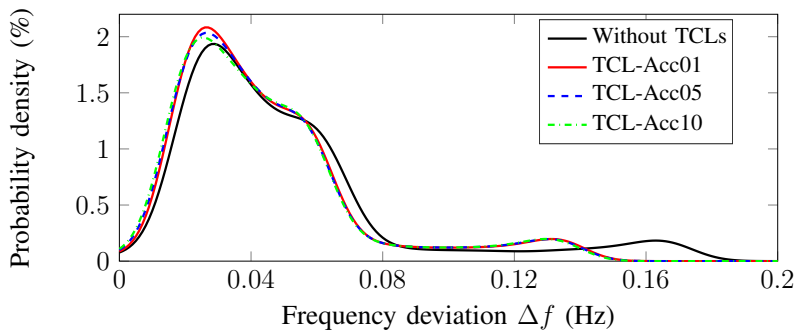

Fig. 14. Frequency responses to an under-frequency event at $t_{\text {event }}=55 \mathrm{~s}$

(a)

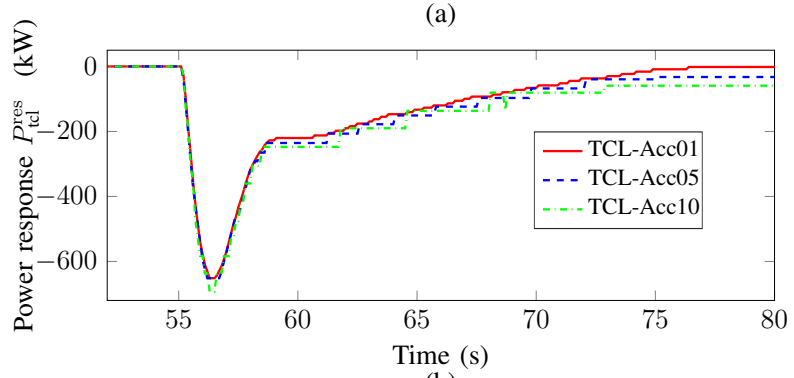

(b)

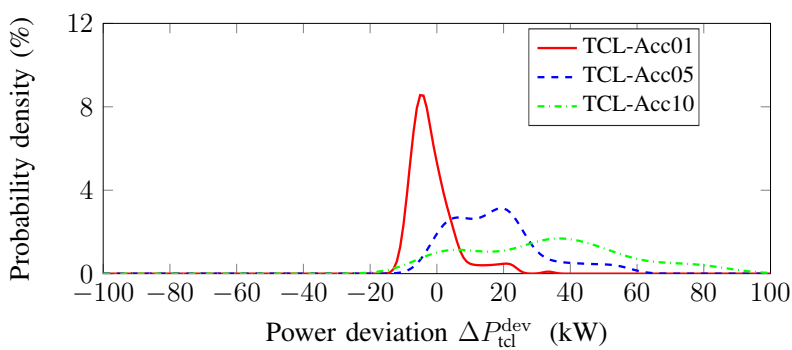

Fig. 15. Power responses of TCLs to under-frequency event at $t_{\text {event }}=55 \mathrm{~s}$

frequency response. The switching activities by the hysteresis control are not included. $n_{\mathrm{tcl}}$ indicates the number of TCLs, which respond to the frequency event. $\eta$ indicates the switching times per TCL, defined by,

$$
\eta=\frac{n_{\text {switch }}}{n_{\text {tcl }}} .
$$

The results of both the conventional method ("CON") and the proposed method ("NEW") are compared. Here, the conventional method means that the ON devices are switched off for the under-frequency event and the OFF devices are switched on for the over-frequency event.

As listed in Table VI, $n_{\text {switch }}$ for both methods are similar. Comparably, $n_{\text {switch }}$ of NEW is a little smaller than that of CON. However, $n_{\mathrm{tcl}}$ of NEW is much larger than that of CON. Accordingly, $\eta$ of NEW is 1 , which implies that the related 
TABLE V

RMSE FOR DIFFERENT SCENARIOS

\begin{tabular}{c|c|c|c}
\hline \hline & $t_{\text {event }}=5 \mathrm{~s}$ & $t_{\text {event }}=55 \mathrm{~s}$ & $t_{\text {event }}=105 \mathrm{~s}$ \\
\hline \hline Acc01 & $12.2 \mathrm{~kW}$ & $12.7 \mathrm{~kW}$ & $13.5 \mathrm{~kW}$ \\
Acc05 & $22.1 \mathrm{~kW}$ & $22.9 \mathrm{~kW}$ & $24.3 \mathrm{~kW}$ \\
Acc10 & $39.9 \mathrm{~kW}$ & $41.8 \mathrm{~kW}$ & $45.3 \mathrm{~kW}$
\end{tabular}

TCL will only do the switching once. Comparably, $\eta$ of CON is 2. It implies that the related TCLs will do the switching twice within a short period, which leads to the short cycling.

TABLE VI

SWITCHING STATISTIC FOR UNDER-FREQUENCY EVENT

\begin{tabular}{c|c|c|c|c|c|c|c}
\hline \hline \multicolumn{2}{c|}{} & \multicolumn{2}{|c|}{$t_{\text {event }}=5 \mathrm{~s}$} & \multicolumn{2}{c|}{$t_{\text {event }}=55 \mathrm{~s}$} & \multicolumn{2}{c}{$t_{\text {event }}=105 \mathrm{~s}$} \\
\cline { 2 - 8 } \multicolumn{2}{c|}{} & CON & NEW & CON & NEW & CON & NEW \\
\hline \hline \multirow{3}{*}{ Acc01 } & $n_{\text {switch }}$ & 190 & 186 & 190 & 186 & 188 & 184 \\
\cline { 2 - 8 } & $n_{\text {tcl }}$ & 95 & 186 & 95 & 186 & 94 & 184 \\
\cline { 2 - 8 } & $\eta$ & 2 & 1 & 2 & 1 & 2 & 1 \\
\hline \multirow{3}{*}{ Acc05 } & $n_{\text {switch }}$ & 190 & 182 & 190 & 182 & 206 & 194 \\
\cline { 2 - 8 } & $n_{\text {tcl }}$ & 95 & 182 & 95 & 182 & 103 & 194 \\
\cline { 2 - 8 } & $\eta$ & 2 & 1 & 2 & 1 & 2 & 1 \\
\hline \multirow{3}{*}{ Acc10 } & $n_{\text {switch }}$ & 210 & 194 & 216 & 200 & 224 & 220 \\
\cline { 2 - 8 } & $n_{\text {tcl }}$ & 105 & 194 & 108 & 200 & 112 & 220 \\
\cline { 2 - 8 } & $\eta$ & 2 & 1 & 2 & 1 & 2 & 1 \\
\hline
\end{tabular}

2) Over-frequency event: Fig. 16 illustrates the frequency response at $t_{\text {event }}=55 \mathrm{~s}$. The frequency rises due to a sudden load loss. Compared to the case without TCLs, the frequency response with TCLs can efficiently decrease the maximum frequency and shorten the recovery time (Fig. 16(a)). According to the probability density curves (Fig. 16(b)), the frequency responses of TCLs with different measurement accuracy levels are almost the same.

(a)

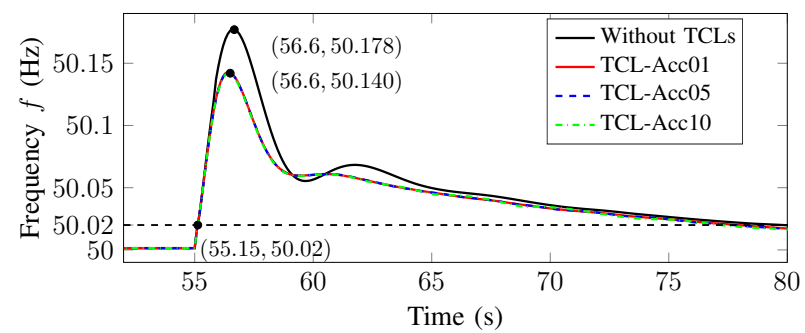

(b)

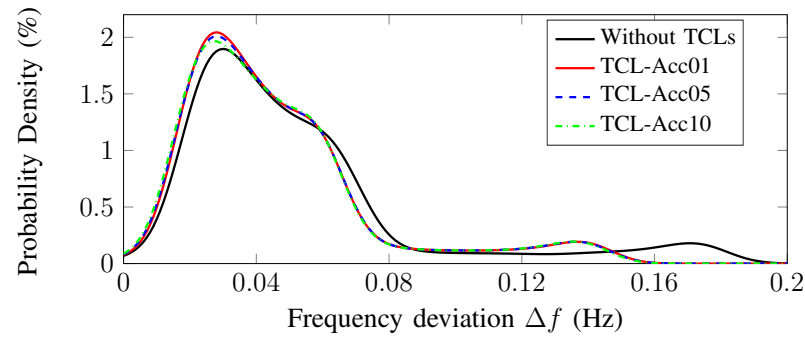

Fig. 16. Frequency responses to a over-frequency event at $t_{\text {event }}=55 \mathrm{~s}$

Fig. 17 illustrates the comparison of aggregated power responses of TCLs $P_{\mathrm{tcl}}^{\mathrm{res}}$. The TCLs with higher measurement

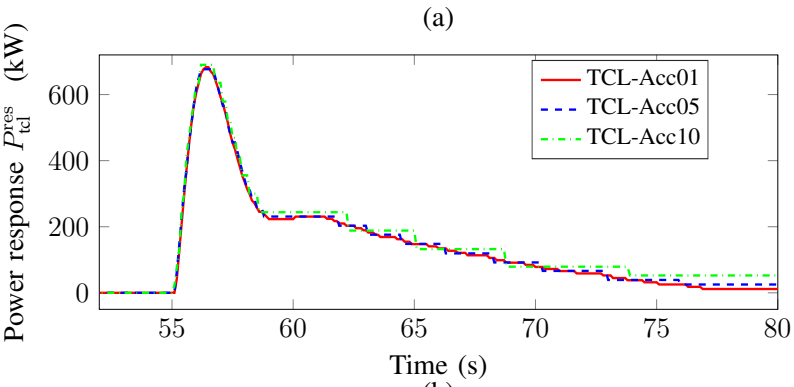

(b)

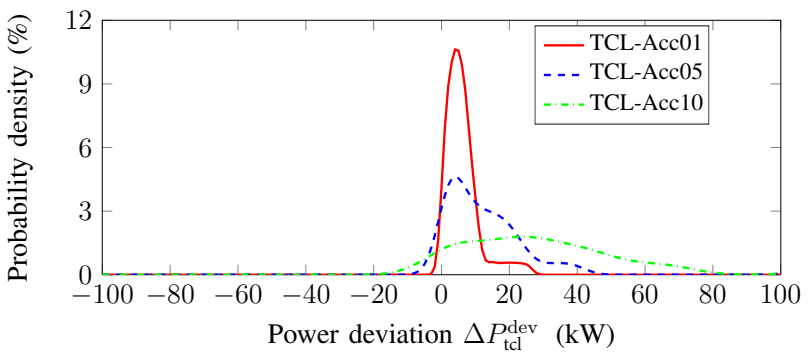

Fig. 17. Power responses of TCLs to over-frequency event at $t_{\text {event }}=55 \mathrm{~s}$

accuracy can track the frequency variation more smoothly (Fig. 17(a)). $\Delta P_{\mathrm{tcl}}^{\mathrm{dev}}$, defined by,

$$
\Delta P_{\mathrm{tcl}}^{\mathrm{dev}}(t)=P_{\mathrm{tcl}}^{\mathrm{res}}(t)-\left(f(t)-\Delta f_{\mathrm{db}}\right) \frac{P_{\mathrm{prim}}^{\mathrm{up}}}{f_{\max }-\Delta f_{\mathrm{db}}},
$$

represents the control error. The TCLs with higher measurement accuracy shows better control performance, as shown in Fig. 17(b).

As listed in Table VII, with the same measurement accuracy, the differences of RMSEs at different event time points are quite small, which verifies the proposed control throughout the whole control period. Moreover, a higher accuracy level leads to a smaller RMSE which implies the control performance is better.

TABLE VII RMSE FOR DIFFERENT SCENARIOS

\begin{tabular}{c|c|c|c}
\hline \hline & $t_{\text {event }}=5 \mathrm{~s}$ & $t_{\text {event }}=55 \mathrm{~s}$ & $t_{\text {event }}=105 \mathrm{~s}$ \\
\hline \hline Acc01 & $12.1 \mathrm{~kW}$ & $12.5 \mathrm{~kW}$ & $12.5 \mathrm{~kW}$ \\
Acc05 & $17.4 \mathrm{~kW}$ & $18.9 \mathrm{~kW}$ & $19.1 \mathrm{~kW}$ \\
Acc10 & $34.3 \mathrm{~kW}$ & $35.9 \mathrm{~kW}$ & $37.0 \mathrm{~kW}$ \\
\hline
\end{tabular}

The switching statistics for the over-frequency event are listed in Table VIII, including $n_{\text {switch }}, n_{\text {tcl }}$, and $\eta$. The results of both the conventional method ("CON") and the proposed method ("NEW") are compared.

As listed in Table VIII, $n_{\text {switch }}$ for both methods are similar. However, $n_{\mathrm{tcl}}$ of NEW is much larger than that of CON. Accordingly, $\eta$ of NEW is 1 , which implies that the related TCL will only do the switching once. Comparably, $\eta$ of CON is 2. It implies that the related TCLs will do the switching twice within a short period, which leads to the short cycling.

\section{CONCLUSION}

In this paper, a hierarchical control scheme is developed to manage a portfolio of TCLs distributed in different areas for 
TABLE VIII

SWITCHING STATISTIC FOR OVER-FREQUENCY EVENT

\begin{tabular}{c|c|c|c|c|c|c|c}
\hline \hline \multicolumn{2}{c|}{} & \multicolumn{2}{|c|}{$t_{\text {event }}=5 \mathrm{~s}$} & \multicolumn{2}{c|}{$t_{\text {event }}=55 \mathrm{~s}$} & \multicolumn{2}{c}{$t_{\text {event }}=105 \mathrm{~s}$} \\
\cline { 2 - 8 } \multicolumn{2}{c|}{} & CON & NEW & CON & NEW & CON & NEW \\
\hline \hline \multirow{3}{*}{ Acc01 } & $n_{\text {switch }}$ & 194 & 196 & 194 & 196 & 198 & 200 \\
\cline { 2 - 8 } & $n_{\text {TCL }}$ & 97 & 196 & 97 & 196 & 99 & 200 \\
\cline { 2 - 8 } & $\eta$ & 2 & 1 & 2 & 1 & 2 & 1 \\
\hline \multirow{3}{*}{ Acc05 } & $n_{\text {switch }}$ & 190 & 190 & 190 & 190 & 206 & 206 \\
\cline { 2 - 8 } & $n_{\text {TCL }}$ & 95 & 190 & 95 & 190 & 103 & 206 \\
\cline { 2 - 8 } & $\eta$ & 2 & 1 & 2 & 1 & 2 & 1 \\
\hline \multirow{3}{*}{ Acc10 } & $n_{\text {switch }}$ & 194 & 190 & 202 & 194 & 194 & 190 \\
\cline { 2 - 8 } & $n_{\text {TCL }}$ & 97 & 190 & 101 & 97 & 97 & 190 \\
\cline { 2 - 8 } & $\eta$ & 2 & 1 & 2 & 1 & 2 & 1 \\
\hline
\end{tabular}

primary frequency control support. The aggregator coordinates multiple distribution substations and proportionally dispatches the primary reserve references. The distribution substation estimates the available power of TCLs for up and down regulations based on the aggregated bin model, and assign the control signals to individual TCLs. With the frequency control loop of the local controller, the TCLs respond to the frequency event autonomously.

The proposed controller can efficiently provide the primary frequency reserve without compromising the users' comfort. The short cycling of TCLs is largely reduced. Besides, due to the autonomous control, the communication requirement is minimized. In the future work, the proposed controller will be implemented in a demand response device and field experiments in the Bornholm system will be carried out.

\section{REFERENCES}

[1] P. Kundur, J. Paserba, V. Ajjarapu, G. Andersson et al., "Definition and classification of power system stability ieee/cigre joint task force on stability terms and definitions," IEEE Trans. Power Syst., vol. 19, no. 3, pp. 1387-1401, 2004.

[2] J. F. Ellison, L. S. Tesfatsion, V. W. Loose, and R. H. Byrne, "Project report: A survey of operating reserve markets in us ISO/RTO-managed electric energy regions," Sandia National Labs Publications, 2012.

[3] ENTSO-E Operation Handbook, "P1-Policy 1: Load-frequency control and performance,", March, 2009.

[4] M. Milligan, P. Donohoo, D. Lew et al., "Operating reserves and wind power integration: an international comparison," in Proc. 9th International Workshop on large-scale integration of wind power into power systems, pp. 18-29, 2010.

[5] K. Moslehi and R. Kumar, "A reliability perspective of the smart grid," IEEE Trans. Smart Grid, vol. 1, no. 1, pp. 57-64, 2010.

[6] J. L. Mathieu, S. Koch, and D. S. Callaway, "State estimation and control of electric loads to manage real-time energy imbalance," IEEE Trans. Power Syst., vol. 28, no. 1, pp. 430-440, 2013.

[7] D. G. Infield, J. Short, C. Home, and L. L. Freris, "Potential for domestic dynamic demand-side management in the UK," in Proc. Power Eng. Soc. Gen. Meeting, 2007, pp. 1-6.

[8] Z. Xu, J. Østergaard, and M. Togeby, "Demand as frequency controlled reserve," IEEE Trans. Power Syst., vol. 26, no. 3, pp. 1062-1071, 2011.

[9] C.-Y. Chang, W. Zhang, J. Lian, and K. Kalsi, "Modeling and control of aggregated air conditioning loads under realistic conditions," in Proc. Innovative Smart Grid Technologies (ISGT), IEEE, 2013, pp. 1-6.

[10] D. S. Callaway and I. A. Hiskens, "Achieving controllability of electric loads," Proc. of the IEEE, vol. 99, no. 1, pp. 184-199, 2011.

[11] E. Vrettos, J. L. Mathieu, and G. Andersson, "Control of thermostatic loads using moving horizon estimation of individual load states," in Proc. Power Systems Computation Conference (PSCC), IEEE, 2014, pp. 1-7.
[12] S. Koch, J. L. Mathieu, and D. S. Callaway, "Modeling and control of aggregated heterogeneous thermostatically controlled loads for ancillary services," in Proc. Power Systems Computation Conference (PSCC), IEEE, 2011, pp. 1-7.

[13] P. J. Douglass, R. Garcia-Valle, P. Nyeng, J. Ostergaard, and M. Togeby, "Smart demand for frequency regulation: Experimental results," IEEE Trans. Smart Grid, vol. 4, no. 3, pp. 1713-1720, 2013.

[14] B. Biegel, L. H. Hansen, P. Andersen, and J. Stoustrup, "Primary control by ON/OFF demand-side devices," IEEE Trans. Smart Grid, vol. 4, no. 4, pp. 2061-2071, 2013.

[15] P. Du and N. Lu, "Appliance commitment for household load scheduling," IEEE Trans. Smart Grid, vol. 2, no. 2, pp. 411-419, 2011.

[16] F. Chen, J. Dai, B. Wang, S. Sahu, M. Naphade, and C.-T. Lu, "Activity analysis based on low sample rate smart meters," in Proc. of the 17th ACM SIGKDD international conference on Knowledge discovery and data mining, 2011, pp. 240-248.

[17] C. Bang, C. B. Rasmussen, and J. Østergaard, "Demand as frequencycontrolled reserve-implementation and practical demonstration programme," Tech. Report, Technical University of Denmark (DTU), EUDP Journalnumber: 64009-0001, 2013.

[18] J. L. Mathieu and D. S. Callaway, "State estimation and control of heterogeneous thermostatically controlled loads for load following," in Proc. 45th Hawaii International Conference on System Science (HICSS), IEEE, 2012, pp. 2002-2011.

[19] S. Soudjani and A. Abate, "Aggregation of thermostatically controlled loads by formal abstractions," in Proc. European Control Conference, 2013, pp. 4232-4237.

[20] R. Durbin, S. Ebby, A. Krogh, and G. Mitchison, "Biological sequence analysis," Cambridge, UK: Cambridge University Press, 1998.

[21] Q. Wu, J. Zimmermann, J. Østergaard, and A. Nielsen, "Hardware-Inthe-Loop (HIL) test of demand as frequency controlled reserve (DFR)." in Proc. International Conference on Advances in Power System Control, Operation and Management (APSCOM), IET, 2015.

[22] D. S. Callaway, "Tapping the energy storage potential in electric loads to deliver load following and regulation, with application to wind energy," Energy Conversion and Management, vol. 50, no. 5, pp. 1389-1400, 2009.

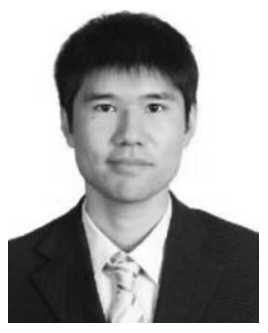

Haoran Zhao (S'12-M'15) received the B.E. degree in electrical engineering and automation from Shandong University, Jinan, China, in 2005, the M.E. degree in electrical engineering and automation from the Technical University of Berlin, Berlin, Germany, in 2009, and the Ph.D. degree in electrical engineering from the Technical University of Denmark, Kgs. Lyngby, Denmark, in 2015.

$\mathrm{He}$ is a Postdoc with the Center for Electric Technology, Technical University of Denmark. He worked as Electrical Engineer with State Grid Corporation of China (SGCC), Beijing, China, in 2005. From August 2010 to September 2011, he worked as Application Developer with DIgSILENT $\mathrm{GmbH}$, Gomaringen, Germany. His research interests include modeling and integration study of wind power, control of energy storage system, and voltage stability analysis. 


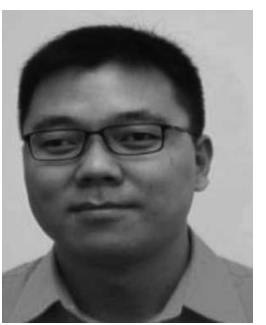

Qiuwei Wu (M'08-SM'15) obtained the B. Eng. and M. Eng. from Nanjing University of Science and Technology, Nanjing, P. R. China, in 2000 and 2003, respectively, both in Power System and Automation. He obtained the PhD degree from Nanyang Technological University, Singapore, in 2009, in Power System Engineering.

He was a senior R\&D engineer with VESTAS Technology R\&D Singapore Pte Ltd from Mar. 2008 to Oct. 2009. He has been working at Department of Electrical Engineering, Technical University of Denmark (DTU) since Nov. 2009 (PostDoc Nov. 2009-Oct. 2010, Assistant Professor Nov. 2010-Aug. 2013, Associate Professor since Sept. 2013). He was a visiting scholar at Department of Industrial Engineering \& Operations Research (IEOR), University of California, Berkeley from Feb. 2012 to May 2012 funded by Ministry of Science, Technology and Innovation, Denmark. $\mathrm{He}$ is also a visiting professor named by Y. Xue, an Academician of Chinese Academy of Engineering, at Shandong University, China.

His research interests are wind power, electric vehicle, demand response, active distribution networks, and real time power system simulation and analysis. He is an Editor of IEEE Transactions on Smart Grid and IEEE Power Engineering Letters. He is also an Associate Editor of International Journal of Electrical Power and Energy Systems.

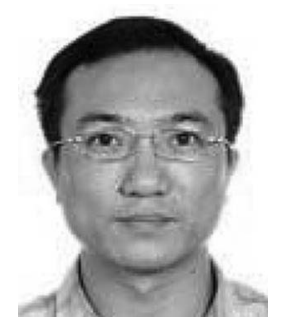

Shaojun Huang (S'13) obtained the B. Eng. from Department of Electrical Engineering, Tsinghua University, Beijing, P. R. China, in 2001. He obtained the MSc from Department of Energy Technology, AAlborg University, Denmark, in 2013. He was a $\mathrm{PhD}$ student at Centre for Electric Power and Energy, Department of Electrical Engineering, Technical University of Denmark from Oct. 2013 to Aug. 2016. Currently, he is PostDoc at Centre for Electric Power and Energy, Department of Electrical Engineering, Technical University of Denmark.

His research interests are congestion management for distribution networks with high penetration of distributed energy resources.

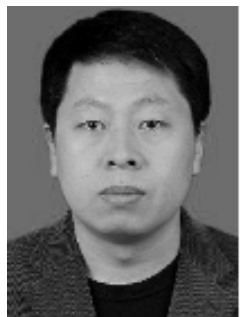

Hengxu Zhang (M'06) received the B.E. degree in Electrical Engineering from Shandong University of Technology, China, in 1998, and the M.S. and Ph.D. degrees in Electrical Engineering from Shandong University, China, in 2000 and 2003, respectively. $\mathrm{He}$ is now a Professor with the School of Electrical Engineering, Shandong University, China.

His main research interests are power system security and stability assessment, power system monitoring, and numerical simulation.

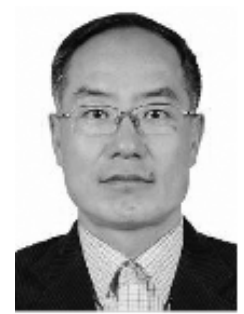

Yutian Liu (SM'96) received the B.E. and M.S. degrees in Electrical Engineering from Shandong University, Jinan, China, in 1984 and 1990, respectively, and the Ph.D. degree in Electrical Engineering from Xi' an Jiaotong University, Xi'an, China, in 1994. $\mathrm{He}$ is currently a Chair Professor with the School of Electrical Engineering, Shandong University, Jinan.

His research interests include power system analysis and control, renewable energy integration, and artificial intelligence application to power systems.

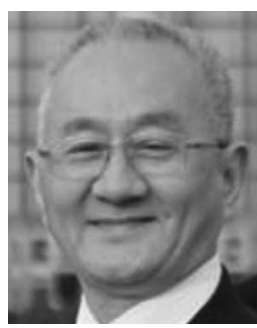

Yusheng Xue (M'88) received the M.Sc. degree in electrical engineering from EPRI, China, in 1981, and the Ph.D. degree in electrical engineering from the University of Liege, Liege, Belgium, in 1987. $\mathrm{He}$ was elected as an Academician of the Chinese Academy of Engineering in 1995.

$\mathrm{He}$ is now the Honorary President of State Grid Electric Power Research Institute (SGEPRI or NARI), China. He holds the positions of Adjunct Professor in many universities in China and is a Conjoint Professor with the University of Newcastle, Callaghan, NSW, Australia. He is also an Honorary Professor with the University of Queensland, Brisbane, Qld., Australia. He has been a member of the PSCC Council, and the Editorin- Chief of Automation of Electric Power System since 1999, and a Member of Editorial Board of IET Generation, Transmission, and Distribution, and Chairman of Technical Committee of Chinese National Committee of CIGRE since 2005. 